\title{
Enantioselective synthesis of planar-chiral 1,11-dioxa[11]paracyclophane- derived phosphoramidites and their use as chiral ligands
}

\author{
Madhurima Hazra, ${ }^{a}$ Kyalo Stephen Kanyiva, ${ }^{b}$ and Takanori Shibata*a,c \\ ${ }^{a}$ Department of Chemistry and Biochemistry, School of Advanced Science and Engineering, Waseda University, Tokyo, 1698050, Japan \\ ${ }^{b}$ International Center for Science and Engineering Programs (ICSEP), Waseda University, 3-4-1 Okubo, Shinjuku, Tokyo 1698555, Japan \\ ${ }^{c}$ JST, ACT-C, 4-1-8 Honcho, Kawaguchi, Saitama 3320012, Japan
}

\section{ARTICLE INFO}

Article history:

Received

Received in revised form

Accepted

Available online

\section{ABSTRACT}

A library of bench-stable planar-chiral 1,11-dioxa[11]paracyclophane-derived phosphoramidites was synthesized by a three step procedure: enantioselective ortholithiation, reductive amination and coupling with phosphorochloridites. The efficacy of these phosphoramidite as chiral ligands was tested in the Pd-catalyzed allylic alkylation of dimethyl malonate and $\mathrm{Cu}$-catalyzed ethylation of chalcone under the reported conditions, and moderate enantioselectivity could be induced.

\section{Introduction}

Since enantiomerically pure compounds play crucial roles in the areas connected to pharmaceuticals, agrochemicals, flavours and fragrance industries, their preparation has become a major goal for synthetic organic chemists. ${ }^{1-3}$ In this context, efficient chiral metal catalysis ${ }^{4}$ is an alluring field exhibiting fruitful results. Innovative design of chiral ligands forms the basis for enantioselectivity. Phosphorus ligands, ${ }^{5-7}$ by far, are widely used as chiral sources. Notable achievements in this field were reported using BINAP $^{8 \mathrm{a}}$ and its derivatives, BINAPO, ${ }^{8 \mathrm{~b}}$ DIPAMP ${ }^{8 c, d}$ and a series of TunaPhos ligands. ${ }^{8 e}$ In spite of immense success with bidentate ligands, last decade has witnessed development of various monodentate chiral phosphorus ligands. Investigation in this area has been comprehensively done by several research groups, such as Feringa, ${ }^{9 \mathrm{a}-\mathrm{b}}$ Pringle, ${ }^{9 \mathrm{c}}$ Reetz, ${ }^{9 \mathrm{~d}}$ and Chan. ${ }^{9 \mathrm{e}}$ Different classes of chiral phosphorus ligands were explored, which play excellent roles in achieving high degree of enantioselectivity in asymmetric synthesis. ${ }^{10-12}$ In this regard, we drew our attention towards the asymmetric synthesis of phosphoramidite ligands due to their easy accessibility, high stability and rapid fine tuning of properties with the variation of substituents at the nitrogen atom. ${ }^{13}$ A variety of asymmetric reactions had been studied with these ligands and reported with significant results, including $\mathrm{Rh}$ - and $\mathrm{Cu}$-catalyzed conjugate additions, ${ }^{14 a, b} \mathrm{Pd}-$ catalyzed allylic alkylation, ${ }^{14 \mathrm{c}}{ }^{15,16}$ hydrogenation, ${ }^{14 \mathrm{~d}}$ hydroformylation, ${ }^{14 \mathrm{e}}$ Heck reaction, ${ }^{14 \mathrm{f}}$ etc. ${ }^{15,16}$
In contrast, we had developed facile synthesis of planar-chiral 1,n-dioxa $[n]$ paracyclophanes through enantioselective ortholithiation. ${ }^{17}$ Due to its unique planar chirality, paracyclophane holds fascinating prospective in terms of asymmetric catalysis. Although the first representative example of cyclophane was developed by Brown and Farthing ${ }^{18}$ in 1949, only one example of [2.2]paracyclophane-derived phosphoramidite ligands was recently published, as far as we know, where they were used as a chiral ligand in the Cu-catalyzed conjugate addition. ${ }^{19}$ Previously, we already reported that planar-chiral 1,ndioxa $[n]$ paracyclophanylphosphines worked as chiral ligands in Pd-catalyzed Sonogashira and Suzuki couplings of limited substrates. $^{20}$ Recently, we further prepared planar-chiral phosphites possessing a 1,n-dioxa[n]paracyclophane scaffold and used them as chiral ligands in Pd-catalyzed allylic alkylation and $\mathrm{Rh}$-catalyzed 1,4 -addition. ${ }^{21}$

In this manuscript, we describe a three-step procedure for the first synthesis of phosphoramidites with planar-chirality on their nitrogen atom via the reaction of planar-chiral 1,11dioxa[11]paracyclophanylamines and 1,11dioxa[11]paracyclophanylmethylamines with 2,2'-biarylene phosphorochloridites derived from chiral or achiral diol and phosphorus trichloride (Scheme 1). Apart from chiral BINOL as a backbone, there are also several reports on $\mathrm{SPINOL}^{22}$ and TADDOL $^{23}$ as a backbone. From this study, thus, a diverse series of ligand library was synthesized bearing planar-chiral paracyclophane scaffold and chiral binol/biphenol as a backbone. The cooperative effect of both planar- and axial chiralities of ligands in inducing enantioselectivity was tested in 
Pd-catalyzed allylic alkylation of dimethyl malonate and $\mathrm{Cu}$ catalyzed conjugate addition of chalcone using diethylzinc.

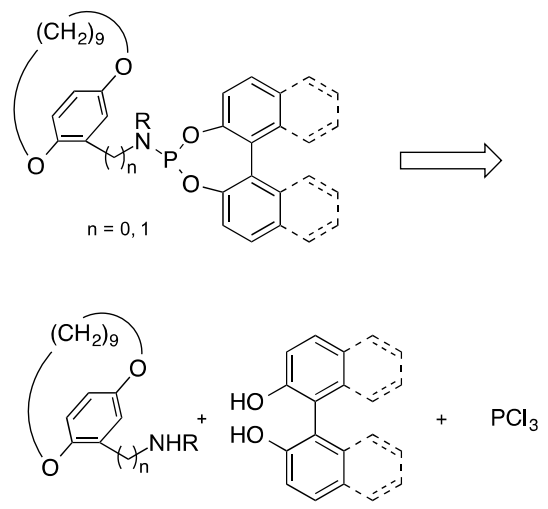

Scheme 1. Creation of a library of planar-chiral phosphoramidites

\section{Results and discussion}

A new family of chiral monodentate phosphoramidite ligands was synthesized in a three-step procedure. First, enantioselective ortho-lithiation ${ }^{17}$ of 1,11 dioxa[11]paracyclophane (1) in presence of $(+)$-sparteine followed by quenching with trimethylsilylmethyl azide ${ }^{24}$ gave planar-chiral paracyclophanylamine $\mathbf{2}$ in excellent ee (Scheme 2).

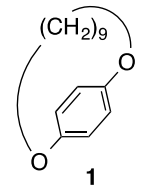

$$
\begin{aligned}
& \text { 1) } \mathrm{sec} \text {-BuLi (2.0 equiv) } \\
& \text { (+)-sparteine ( } 1.0 \text { equiv) } \\
& \mathrm{Et}_{2} \mathrm{O},-78^{\circ} \mathrm{C}, 2 \mathrm{~h} \\
& \hline \text { 2) } \mathrm{TMSCH}_{2} \mathrm{~N}_{3}(5.0 \text { equiv) } \\
& \mathrm{Et}_{2} \mathrm{O},-78^{\circ} \mathrm{C}-\mathrm{rt}
\end{aligned}
$$

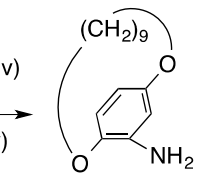

2: $60 \%, 98 \%$ ee
Scheme 2. Enantioselective synthesis of a planar-chiral paracyclophanylamine

Next, reductive amination of carbonyl compounds by planarchiral primary amine $\mathbf{2}$ using sodium triacetoxyborohydride gave secondary paracyclophanylamines 3a-3c (Eq. 1 in Scheme 3). On the other hand, reductive amination of planar-chiral aldehyde $4^{17}$ by benzyl- and isopropylamine under the same reaction conditions gave secondary paracyclophanylmethylamines $\mathbf{5 a}$ and 5b (Eq. 2 in Scheme 3). The retention of enantiomeric excess through the reductive amination was ascertained by compounds $3 \mathbf{a}$ and $\mathbf{5 a}$.

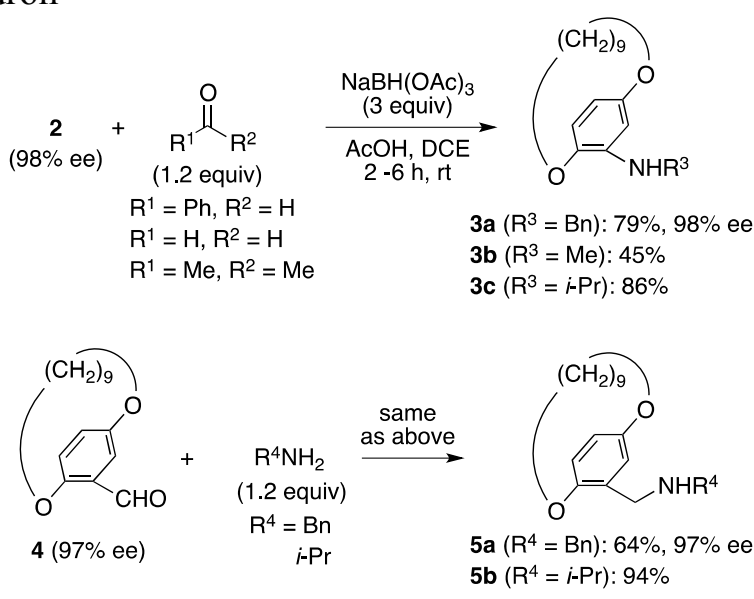

Scheme 3. Synthesis of planar-chiral secondary amines

Finally, these obtained secondary amines were lithiated using $n$-butyllithium in presence of tetramethylethylenediamine (TMEDA), and subsequently treated with in situ prepared 2,2'biarylene phosphorochloridites (Scheme 4). When paracyclophanylamine 3a was reacted with axially chiral 2,2'binaphthyl phosphorochloridite, $\left(R_{\mathrm{a}}\right)-\mathbf{L} \mathbf{1}$ and $\left(S_{\mathrm{a}}\right)-\mathbf{L} \mathbf{1}$ were obtained respectively, both which have planar and axial chiralities. From paracyclophanylmethylamine 5a, $\left(R_{\mathrm{a}}\right)-\mathbf{L} \mathbf{2}$ and $\left(S_{\mathrm{a}}\right)$-L2 were obtained as diastereomers. $N$-Methyl and $N$ isopropyl analogues $\left(R_{\mathrm{a}}\right)$ - $\mathbf{L 3}$ and $\left(R_{\mathrm{a}}\right)$ - $\mathbf{L} \mathbf{4}$ could be prepared from $\mathbf{3 b}$ and 3c, respectively. The reaction of achiral 2,2'-biphenylene phosphorochloridite with 3a, 5a, and 5b gave L5-L7. Tetramethyl-substituted biphenylene-derived ligands L8 and L9 were also prepared using the same protocol. All these phosphoramidites were purified as solids by preparative chromatography of silica gel and were stable at ambient conditions, thus, they could easily be applied to asymmetric reactions as chiral ligands due to ease in handling. 

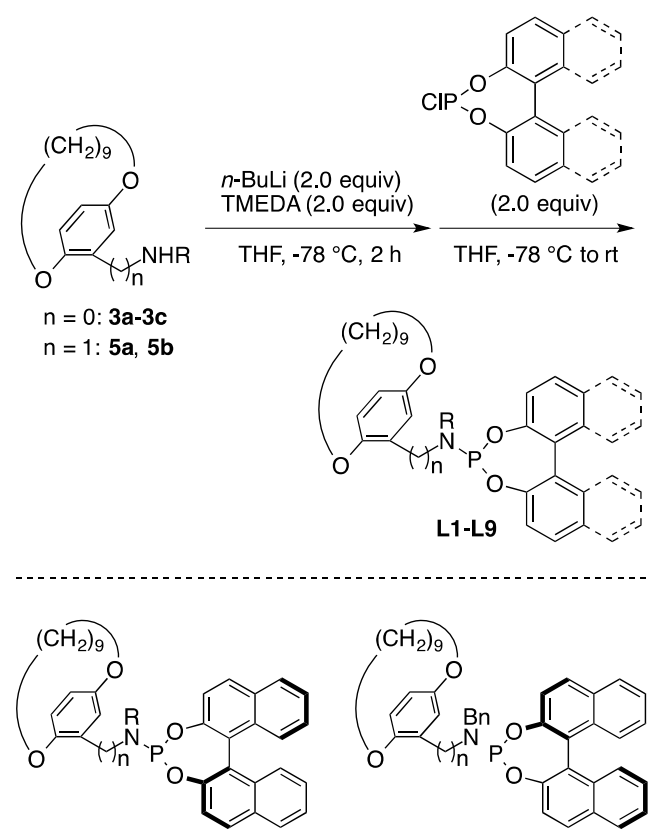

$\left(R_{\mathrm{a}}\right) \mathrm{-L} 1(\mathrm{n}=0, \mathrm{R}=\mathrm{Bn}): 41 \%$ $\left(R_{\mathrm{a}}\right)$-L2 $(\mathrm{n}=1, \mathrm{R}=\mathrm{Bn}): 36 \%$ $\left(R_{\mathrm{a}}\right)$-L3 $(\mathrm{n}=0, \mathrm{R}=\mathrm{Me}): 30 \%$

$\left(R_{\mathrm{a}}\right)$-L4 ( $\left.\mathrm{n}=0, \mathrm{R}=i-\mathrm{Pr}\right): 20 \%$

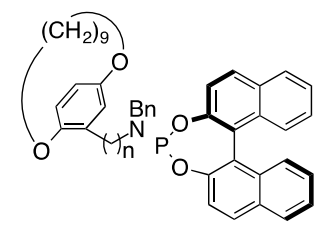

$\left(S_{\mathrm{a}}\right)$-L1 $(\mathrm{n}=0): 65 \%$ $\left(S_{\mathrm{a}}\right)$-L2 $(\mathrm{n}=1): 45 \%$

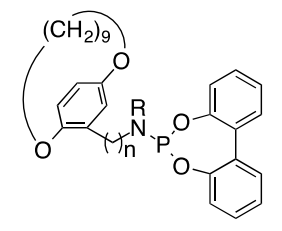

$\mathbf{L 5}(\mathrm{n}=0, \mathbf{R}=\mathrm{Bn}): \mathbf{7 0 \%}$ L6 $(n=1, R=B n): 47 \%$ L7 $(\mathrm{n}=1, \mathrm{R}=i-\mathrm{Pr}): 30 \%$

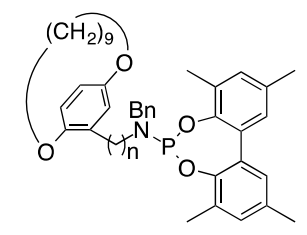

L8 $(\mathrm{n}=0): 55 \%$ L9 $(n=1): 39 \%$

Scheme 4. Synthesis of planar-chiral phosphoramidites

To test the efficacy of our originally developed planar-chiral paracyclophane-derived phosphoramidite ligands L1-L9, we first chose Pd-catalyzed asymmetric allylic alkylation, and performed the reaction under the reported literature conditions without further optimization (Table 1$){ }^{25}$ The reaction of dimethyl malonate 7 with (E)-1,3-diphenylallyl acetate $\mathbf{6}$ furnished the allylated product $\mathbf{8}$ with low to excellent yield. Stereoselectivity and the enantioselectivity varied depending on the structure of the ligands. The diastereomeric pair of $\left(R_{\mathrm{a}}\right)-\mathbf{L} 1$ and $\left(S_{\mathrm{a}}\right)$-L1 possessing both planar and axial chiralities gave $(R)$ 8 as a major enantiomer with moderate ee, which suggests that stereoselectivity was mainly controlled by planar chirality of paracyclophane scaffold (entries 1 and 2). However, BINOL moiety was also significant for enantioinduction because phosphoramidite ligand $\mathbf{L 5}$ and $\mathbf{L 8}$ derived from 2,2'-biphenol and tetramethyl-substituted 2,2'-biphenol resulted in low ee (entries 7 and 10). In contrast, the diastereomeric pair of paracyclophanylmethylamine-based $\left(R_{\mathrm{a}}\right)-\mathbf{L} \mathbf{2}$ and $\left(S_{\mathrm{a}}\right)-\mathbf{L} \mathbf{2}$ showed opposite stereoselectivity, which suggests that the stereoselectivity was mainly controlled by axial chirality of the BINOL moiety (entries 3 and 4). Surprisingly, however, ligand L6 possessing only planar chirality realized the best ee of $70 \%$ (entry 8). Other paracyclophanylmethylamine-based ligands L7 and $\mathbf{L 9}$ gave poorer enantioselectivities (entries 9 and 11). These results show that planar-chirality of dioxaparacyclophane operated as an efficient chiral scaffold in phosphoramidite ligand, but the choice of substituent on the nitrogen atom and 2,2'-biphenylene diol moiety was also critical.

Table 1

Enantioselective Pd-catalyzed allylic alkylation of malonate

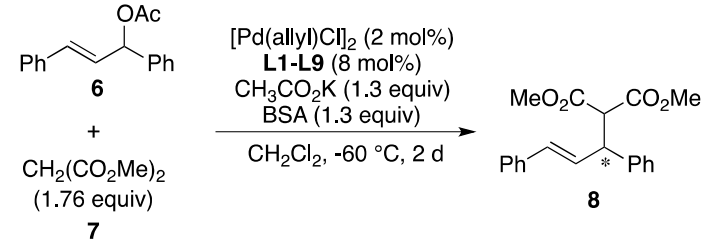

\begin{tabular}{cccc}
\hline Entry & Ligand & ${\text { Yield }(\%)^{\mathrm{a}}}$ & Ee $(\%)^{\mathrm{b}}$ \\
\hline 1 & $\left(R_{\mathrm{a}}\right)-\mathbf{L 1}$ & 49 & $48(R)$ \\
2 & $\left(S_{\mathrm{a}}\right)-\mathbf{L 1}$ & 76 & $39(R)$ \\
3 & $\left(R_{\mathrm{a}}\right)-\mathbf{L 2}$ & 75 & $62(S)$ \\
4 & $\left(S_{\mathrm{a}}\right)-\mathbf{L 2}$ & 74 & $67(R)$ \\
5 & $\left(R_{\mathrm{a}}\right)-\mathbf{L 3}$ & 81 & $12(S)$ \\
6 & $\left(R_{\mathrm{a}}\right)-\mathbf{L 4}$ & 36 & $10(R)$ \\
7 & $\mathbf{L 5}$ & 97 & $6(R)$ \\
8 & $\mathbf{L 6}$ & 82 & $\mathbf{7 0}(R)$ \\
9 & $\mathbf{L 7}$ & 86 & $51(R)$ \\
10 & $\mathbf{L 8}$ & 23 & $15(R)$ \\
11 & $\mathbf{L 9}$ & 50 & $8(R)$ \\
\hline
\end{tabular}

${ }^{\mathrm{a}}$ Isolated yield. ${ }^{\mathrm{b}}$ Ee was determined by HPLC analysis using Daicel Chiralcel OD-3 (eluent: 1\% 2-propanol in hexane, flow rate: $0.5 \mathrm{~mL} / \mathrm{min}$ ). The absolute configuration of the major isomer was described in the parentheses.

Next, we examined these ligands L1-L9 for Cu-catalyzed asymmetric conjugate addition of chalcone (9) with diethylzinc under the literature condition, ${ }^{26}$ and the alkylated product $\mathbf{1 0}$ was obtained in moderate to quantitative yield (Table 2). In contrast to the Pd-catalyzed allylic alkylation, the diastereomeric pair of $\left(R_{\mathrm{a}}\right)$-L1 and $\left(S_{\mathrm{a}}\right)$-L1 gave the opposite enantiomers of ethylated product 10, and the ee was quite different (entries 1 and 2). The diastereomeric pair of paracyclophanylmethylamine-based $\left(R_{\mathrm{a}}\right)$ $\mathbf{L 2}$ and $\left(S_{\mathrm{a}}\right)$-L2 showed low enantioselectivity (entries 3 and 4). The choice of substituent on the nitrogen atom was important, and methyl group gave the best ee of $64 \%$ among paracyclophanylamine-based ligands possessing a BINOL scaffold (entries 1, 5 and 6). ${ }^{27}$ As for the ligand having an achiral 2,2'-biphenol scaffold, isopropyl group on the nitrogen atom could induce moderate ee (entries 5-9). Thus, a slight alteration in the structure of phosphoramidite ligands resulted in the different degree of enantioinduction by the cooperative effect of three substituents of the nitrogen atom. Actually, when $\left(R_{\mathrm{a}}\right)-\mathbf{L} \mathbf{1 0}$, which is a planar chirality-free analogue of $\left(R_{\mathrm{a}}\right)-\mathbf{L} \mathbf{3}$, was used, the enantioselectivity was dramatically decreased (Scheme 5 ). 
Table 2

Enantioselective $\mathrm{Cu}$-catalyzed conjugate addition of chalcone with diethylzinc

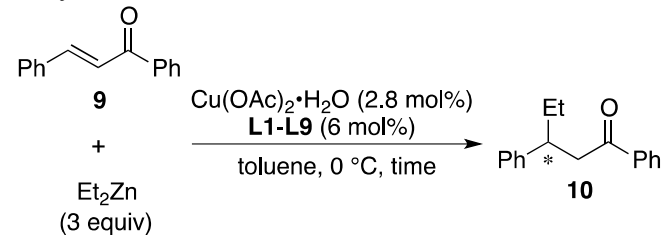

\begin{tabular}{ccccc}
\hline Entry & Ligand & Time $(\mathrm{h})$ & ${\text { Yield }(\%)^{\mathrm{a}}}$ & Ee $(\%)^{\mathrm{b}}$ \\
\hline 1 & $\left(R_{\mathrm{a}}\right)-\mathbf{L} 1$ & 1.5 & $>99$ & $44(R)$ \\
2 & $\left(S_{\mathrm{a}}\right)-\mathbf{L} 1$ & 1.0 & $>99$ & $18(S)$ \\
3 & $\left(R_{\mathrm{a}}\right)-\mathbf{L} 2$ & 3.0 & $>99$ & $6(S)$ \\
4 & $\left(S_{\mathrm{a}}\right)-\mathbf{L} \mathbf{2}$ & 1.0 & $>99$ & $17(R)$ \\
5 & $\left(R_{\mathrm{a}}\right)-\mathbf{L 3}$ & 1.5 & 91 & $\mathbf{6 4}(R)$ \\
6 & $\left(R_{\mathrm{a}}\right)-\mathbf{L} 4$ & 2.0 & $>99$ & $7(S)$ \\
7 & $\mathbf{L 5}$ & 3.0 & $>99$ & $4(R)$ \\
8 & $\mathbf{L 6}$ & 5.5 & 70 & $19(R)$ \\
9 & $\mathbf{L 7}$ & 1.5 & 89 & $50(R)$ \\
10 & $\mathbf{L 8}$ & 5.5 & 88 & $14(R)$ \\
11 & $\mathbf{L 9}$ & 5.5 & 63 & $4(R)$
\end{tabular}

${ }^{\mathrm{a}}$ Isolated yield. ${ }^{\mathrm{b}}$ Ee was determined by HPLC analysis using Daicel Chiralcel OD-3 (eluent: 0.5\% 2-propanol in hexane, flow rate: $1.0 \mathrm{~mL} / \mathrm{min}$ ). The absolute configuration of the major isomer was described in the parentheses.

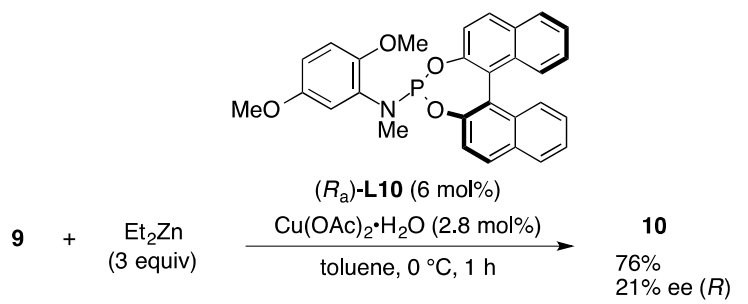

Scheme 5. Control experiment using $\left(R_{\mathrm{a}}\right)$-L10 having no planar chirality

\section{Conclusions}

A library of chiral phosphoramidite ligands with planar chirality on their nitrogen atom was synthesized and tested as chiral ligands in two well-known asymmetric reactions under the literature conditions. These newly synthesized ligands are highly air- and moisture-stable and thus, stringent care is not required to handle them. A slight tuning of ligand structure effects a diverse change of enantioinduction in Pd-catalyzed allylic alkylation and $\mathrm{Cu}$-catalyzed conjugate addition reactions. Applications of these phosphoramidite ligands possessing a planar-chiral paracyclophane scaffold to other asymmetric reactions are on going in our laboratory.

\section{Experimental section}

\subsection{General}

Unless otherwise noted, all materials were purchased from commercial suppliers and used as received. Anhydrous solvents were stocked on activated molecular sieves $4 \AA$ under argon atmosphere, and degassed by argon bubbling prior to use. All reactions were carried out under argon atmosphere in oven-dried glassware with a magnetic stirring bar.
${ }^{1} \mathrm{H}$ NMR spectra were recorded on JEOL AL-400 (400 MHz) spectrometers. The chemical shifts were reported in parts per million $(\delta)$ relative to internal standard TMS $(0 \mathrm{ppm})$ for $\mathrm{CDCl}_{3}$. The peak patterns are indicated as follows: s, singlet; $d$, doublet; $\mathrm{dd}$, doublet of doublet; $\mathrm{t}$, triplet; q, quartet; m, multiplet, brs, broad singlet. The coupling constants, $J$, are reported in Hertz (Hz). ${ }^{13} \mathrm{C}$ NMR spectra were obtained by JEOL AL-400 (100 $\mathrm{MHz}$ ) spectrometers and referenced to the internal solvent signals (central peak is $77.0 \mathrm{ppm}$ in $\left.\mathrm{CDCl}_{3}\right) .{ }^{31} \mathrm{P}$ NMR spectra were obtained by JEOL AL-400 (200 MHz) spectrometers and $\mathrm{H} 3 \mathrm{PO} 4$ was used as an external standard $(0.00 \mathrm{ppm}) . \mathrm{CDCl}_{3}$ was used as a NMR solvent. High-resolution mass spectra (HRMS) were measured on ESI (Electrospray ionization) method at a JEOL GC-mate II. Preparative thin-layer chromatography (PTLC) was performed with silica gel-precoated glass plates (Merck 60 GF254) prepared in our laboratory. Flash column chromatography was performed over silica gel 200-300.

\subsection{Experimental procedure for the preparation of amine 2 (Scheme 2)}

In a two-neck flask purged with argon, a solution of 1,11dioxa[11]paracyclophane (1) $(0.5 \mathrm{mmol})$ in $\mathrm{Et}_{2} \mathrm{O}(0.5 \mathrm{ml})$ was added followed by $(+)$-sparteine ( 1 equiv) and the resulting solution was cooled to $-78^{\circ} \mathrm{C}$. sec-BuLi $(0.98 \mathrm{M})$ (2 equiv) was added dropwise and stirred at $-78{ }^{\circ} \mathrm{C}$ for $2 \mathrm{~h}$. A solution of trimethylsilylmethyl azide (5 equiv) in $\mathrm{Et}_{2} \mathrm{O}(0.6 \mathrm{~mL})$ was added dropwise and the reaction mixture was warmed to ambient temperature. The reaction was quenched with saturated aqueous solution of $\mathrm{NH}_{4} \mathrm{Cl}$ and extracted with EtOAc $(10 \mathrm{ml} \times 3)$. The organic layer was washed with brine and dried with $\mathrm{Na}_{2} \mathrm{SO}_{4}$. The solution was evaporated and purified through PTLC (eluent: hexane/EtOAc = 5/1). Yield: $75.8 \mathrm{mg}, 60 \%$.

\subsubsection{3-Amino-1,11-dioxa[11]paracyclophane} (2).

Yellow viscous liquid. ${ }^{1} \mathrm{H}$ NMR (ppm) $\delta$ 6.90-6.88 (m, 1H), 6.45-6.43 (m, 2H), 4.26-4.15 (m, 4H), 3.87 (brs, 2H), 1.71-1.61 $(\mathrm{m}, 4 \mathrm{H}), 1.03-0.90(\mathrm{~m}, 10 \mathrm{H}) ;{ }^{13} \mathrm{C} \mathrm{NMR}(\mathrm{ppm}) \delta 155.7,141.5$, $140.0,120.6,110.0,108.2,71.4,71.3,30.2,30.0,27.9,26.1$, 26.1, 25.7, 25.4; IR ( $\left.\mathrm{cm}^{-1}\right) 3365,2924,2851,2362,1617,1506$, 1457, 1198, 1163, 982; HRMS(ESI) calcd for $\mathrm{C}_{15} \mathrm{H}_{24} \mathrm{NO}_{2}$ $[\mathrm{M}+\mathrm{H}]^{+}:$250.1802; found: $250.1802 .[\alpha]_{\mathrm{D}}^{23}=+38.6(c \quad 0.57$, $\mathrm{CHCl}_{3}, 98 \%$ ee). Ee was determined by HPLC analysis using a chiral column (Daicel Chiralpak IA: 4 x $250 \mathrm{~mm}, 254 \mathrm{~nm}$ UV detector, rt, eluent: $40 \%$ EtOAc in hexane, flow rate: 0.5 $\mathrm{mL} / \mathrm{min}$, retention time: $10.2 \mathrm{~min}$ for minor isomer and $11.0 \mathrm{~min}$ for major isomer).

\subsection{General experimental procedure for reductive amination (Eq. 1 in Scheme 3)}

To a two-neck flask purged with argon, a solution of appropriate amine $(0.1 \mathrm{mmol})$ and aldehyde (1.2 equiv) in 1,2dichloroethane $(0.3 \mathrm{~mL})$ was added followed by $\mathrm{NaBH}(\mathrm{OAc})_{3}(3$ equiv) and $\mathrm{AcOH}(0.02 \mathrm{~mL})$. The reaction mixture was stirred till the completion of reaction. A saturated aqueous solution of $\mathrm{K}_{2} \mathrm{CO}_{3}$ was added to the reaction mixture and extracted with dichloromethane $(10 \mathrm{ml} \times 3)$. The organic layer was washed with water, dried over $\mathrm{Na}_{2} \mathrm{SO}_{4}$ and evaporated to dryness. The obtained crude products were isolated through PTLC.

\subsubsection{3-Benzylamino-1,11-} dioxa[11]paracyclophane (3a)

Yellow viscous oil. PTLC (eluent: hexane/EtOAc $=5 / 1$ ). Yield: $12.5 \mathrm{mg}, 79 \%$. ${ }^{1} \mathrm{H}$ NMR (ppm) $\delta$ 7.40-7.30 (m, 4H), 7.25-7.23 $(\mathrm{m}, 1 \mathrm{H}), 6.84(\mathrm{dd}, J=4.8,3.8 \mathrm{~Hz}, 1 \mathrm{H}), 6.34-6.32(\mathrm{~m}, 1 \mathrm{H}), 6.28$ $6.27(\mathrm{~m}, 1 \mathrm{H}), 4.77$ (brs, 1H), 4.34-4.12 (m, 4H), 4.06-4.04 (m, 
$2 \mathrm{H}), 1.61-1.47(\mathrm{~m}, 4 \mathrm{H}), 0.95-0.74(\mathrm{~m}, 10 \mathrm{H}) ;{ }^{13} \mathrm{C}$ NMR $(\mathrm{ppm}) \delta$ $155.9,141.9,141.1,139.5,128.5,127.4,127.1,119.4,107.8$, 104.3, 71.6, 71.4, 47.9, 30.2, 30.1, 27.9, 26.2, 26.1, 25.8, 25.6 (two pair of peaks are overlapped at aromatic region); IR $\left(\mathrm{cm}^{-1}\right)$ 2924, 2852, 2362, 2340, 1603, 1516, 1456, 1182, 977, 697,419; HRMS(ESI) calcd for $\mathrm{C}_{22} \mathrm{H}_{29} \mathrm{NNaO}_{2}[\mathrm{M}+\mathrm{Na}]^{+}: 362.2090$; found: 362.2091. $[\alpha]_{\mathrm{D}}^{25}=+63.2$ (c $0.76, \mathrm{CHCl}_{3}, 98 \%$ ee). Ee was determined by HPLC analysis using a chiral column (Daicel Chiralpak IA: 4 x 250mm, 254nm UV detector, rt, eluent: $10 \%$ EtOAc in hexane, flow rate: $1 \mathrm{~mL} / \mathrm{min}$, retention time: $8.3 \mathrm{~min}$ for minor isomer and $10.7 \mathrm{~min}$ for major isomer).

4.3.2. 13-Methylamino-1,11dioxa[11]paracyclophane $(3 b)$

Yellow viscous oil. PTLC (eluent: hexane/EtOAc $=5 / 1$ ). Yield: $11.7 \mathrm{mg}, 45 \%$. ${ }^{1} \mathrm{H}$ NMR (ppm) $\delta$ 6.90-6.87 (m, 1H), 6.40-6.35 (m, 2H), 4.34 (brs, $1 \mathrm{H}), 4.26-4.15(\mathrm{~m}, 4 \mathrm{H}), 2.88$ (d, $J=2.5 \mathrm{~Hz}$, $3 \mathrm{H}), 1.69-1.61(\mathrm{~m}, 4 \mathrm{H}), 1.04-0.90(\mathrm{~m}, 10 \mathrm{H}) ;{ }^{13} \mathrm{C}$ NMR $(\mathrm{ppm}) \delta$ 156.1, 143.4, 141.3, 119.3, 107.3, 103.6, 71.6, 71.4, 30.5, 30.3, 30.0, 28.0, 26.1, 26.1, 25.8, 25.4; IR $\left(\mathrm{cm}^{-1}\right)$ 3426, 2923, 2852, 2362, 1606, 1518, 1457, 1186, 981, 447, 419; HRMS(ESI) calcd for $\mathrm{C}_{16} \mathrm{H}_{26} \mathrm{NO}_{2}[\mathrm{M}+\mathrm{H}]^{+}$: 264.1958; found: 264.1958. $[\alpha]_{\mathrm{D}}^{26}=$ $+88.2\left(\right.$ c 1.32, $\left.\mathrm{CHCl}_{3}\right)$.

\subsubsection{3-Isopropylamino-1,11- dioxa[11]paracyclophane $(3 \mathrm{c})$}

Pale yellow viscous oil. PTLC (eluent: hexane/EtOAc $=5 /$ ). Yield: $24.8 \mathrm{mg}, 86 \%$. ${ }^{1} \mathrm{H}$ NMR (ppm) $\delta 6.85-6.83(\mathrm{~m}, 1 \mathrm{H}), 6.32-$ $6.30(\mathrm{~m}, 2 \mathrm{H}), 4.24-4.10(\mathrm{~m}, 5 \mathrm{H}), 3.57$ (brs, $1 \mathrm{H}), 1.65-1.60(\mathrm{~m}$, $4 \mathrm{H}), 1.25-1.20(\mathrm{~m}, 6 \mathrm{H}), 1.03-0.87(\mathrm{~m}, 10 \mathrm{H}) ;{ }^{13} \mathrm{C}$ NMR $(\mathrm{ppm}) \delta$ 156.0, 141.3, 141.0, 119.5, 106.8, 104.0, 71.6, 71.4, 43.6, 30.4, $30.1,27.9,26.1,26.1,25.8,25.4,23.0,22.9$; IR $\left(\mathrm{cm}^{-1}\right) 3419$, 2924, 2853, 2363, 1605, 1516, 1458, 1187, 420; HRMS(ESI) calcd for $\mathrm{C}_{18} \mathrm{H}_{30} \mathrm{NO}_{2}[\mathrm{M}+\mathrm{H}]^{+}:$292.2270; found: 292.2271. $[\alpha]_{\mathrm{D}}^{25}$ $=+98.4\left(c 0.57, \mathrm{CHCl}_{3}\right)$.

\subsubsection{3-(Benzylamino)methyl-1,11- dioxa[11]paracyclophane (5a)}

Pale yellow viscous oil. PTLC (eluent: hexane/EtOAc $=3 / 1$ ). Yield: $21.5 \mathrm{mg}, 64 \%$. ${ }^{1} \mathrm{H}$ NMR (ppm) $\delta 7.35-7.30$ (m, 4H), 7.26$7.24(\mathrm{~m}, 1 \mathrm{H}), 7.02-7.00(\mathrm{~m}, 1 \mathrm{H}), 6.99-6.94(\mathrm{~m}, 2 \mathrm{H}), 4.22-4.17$ $(\mathrm{m}, 4 \mathrm{H}), 4.06(\mathrm{~d}, J=13.7 \mathrm{~Hz}, 1 \mathrm{H}), 3.83-3.74(\mathrm{~m}, 2 \mathrm{H}), 3.63(\mathrm{~d}, J$ $=13.7 \mathrm{~Hz}, 1 \mathrm{H}), 2.02(\mathrm{brs}, 1 \mathrm{H}), 1.68-1.58(\mathrm{~m}, 4 \mathrm{H}), 0.96-0.73(\mathrm{~m}$, $10 \mathrm{H}) ;{ }^{13} \mathrm{C}$ NMR (ppm) $\delta 154.5,152.6,140.3,133.0,128.3$, 128.1, 126.8, 121.6, 120.0, 119.7, 71.7, 71.1, 52.9, 48.5, 30.0, $29.8,27.7,26.2,26.0,25.8,25.2$ (two pairs of peaks are overlapped at aromatic region); IR $\left(\mathrm{cm}^{-1}\right)$ 2925, 2854, 2361, 1491, 1456, 1199, 1150, 979, 824, 736, 698; HRMS(ESI) calcd for $\mathrm{C}_{23} \mathrm{H}_{32} \mathrm{NO}_{2}[\mathrm{M}+\mathrm{H}]^{+}$: 354.2425; found: 354.2428. [ $\left.\alpha\right]_{\mathrm{D}}^{26}=$ +13.0 ( $c 0.85, \mathrm{CHCl}_{3}, 97 \%$ ee). Ee was determined by HPLC analysis using a chiral column (Daicel Chiralcel OD-H: $4 \mathrm{x}$ $250 \mathrm{~mm}, 254 \mathrm{~nm}$ UV detector, rt, eluent: 10\% 2-propanol in hexane, flow rate: $0.5 \mathrm{~mL} / \mathrm{min}$, retention time: $16.4 \mathrm{~min}$ for major isomer and $24.0 \mathrm{~min}$ for minor isomer).

\subsubsection{3-(Isopropylamino)methyl-1,11- dioxa[11]paracyclophane $(5 b)$}

Yellow viscous oil. PTLC (eluent: Hex/EtOAc $=3 / 1$ ). Yield: $32.1 \mathrm{mg}, 94 \% .{ }^{1} \mathrm{H}$ NMR (ppm) $\delta 6.97(\mathrm{~d}, J=2.4 \mathrm{~Hz}, 2 \mathrm{H}), 6.94$ $6.92(\mathrm{~m}, 1 \mathrm{H}), 4.22-4.17(\mathrm{~m}, 4 \mathrm{H}), 4.03(\mathrm{~d}, J=13.5 \mathrm{~Hz}, 1 \mathrm{H}), 3.61$ $(\mathrm{d}, J=13.5 \mathrm{~Hz}, 1 \mathrm{H}), 2.79-2.77(\mathrm{dd}, J=5.9,2.7 \mathrm{~Hz}, 1 \mathrm{H}), 1.66-$ $1.61(\mathrm{~m}, 4 \mathrm{H}), 1.43$ (brs, $1 \mathrm{H}), 1.07$ (d, $J=5.6 \mathrm{~Hz}, 6 \mathrm{H}), 0.97-0.67$ $(\mathrm{m}, 10 \mathrm{H}) ;{ }^{13} \mathrm{C}$ NMR $(\mathrm{ppm}) \delta 154.5,152.6,133.5,121.5,119.9$, 119.5, 71.7, 71.1, 47.4, 46.7, 30.1, 29.9, 27.8, 26.2, 26.0, 25.8, 25.3, 23.0, 22.7. IR $\left(\mathrm{cm}^{-1}\right) 3853,3749,3735,3649,2924,2853$, 2361, 1490, 1457, 1193; HRMS(ESI) calcd for $\mathrm{C}_{19} \mathrm{H}_{32} \mathrm{NO}_{2}$
$[\mathrm{M}+\mathrm{H}]^{+}:$306.2425; found: 306.2428. $[\alpha]_{\mathrm{D}}^{28}=+8.8$ (c 0.61 , $\mathrm{CHCl}_{3}$ ).

4.4. General procedure for the synthesis of phosphoramidite ligands (L1-L9) (Scheme 4)

In a Schlenk tube evacuated and purged with argon ( 3 times), a solution of appropriate planar-chiral amine (3a-3c, 5a and $\mathbf{5 b}$ ) $(0.1 \mathrm{mmol})$ in THF $(0.5 \mathrm{~mL})$ and TMEDA ( 2 equiv) were added and cooled to $-78{ }^{\circ} \mathrm{C}$. A hexane solution of $n$-BuLi $(1.54 \mathrm{M}, 2$ equiv) was added dropwise to the solution, and the reaction mixture was stirred for $2 \mathrm{~h}$ at $-78^{\circ} \mathrm{C}$. A THF solution $(0.6 \mathrm{~mL})$ of commercially available chlorodioxaphosphepin (2 equiv) or in situ prepared 2,2'-biarylene phosphorochloridite [prepared by mixing the corresponding biaryl diol $(0.20 \mathrm{mmol})$ and $\mathrm{PCl}_{3}(0.28$ mmol) at $75{ }^{\circ} \mathrm{C}$ overnight] was added dropwise at $-78{ }^{\circ} \mathrm{C}$ and warmed to room temperature overnight. The resulting solution was filtered through filter paper using dichloromethane and was evaporated to dryness. The crude products were purified through PTLC to furnish desired ligands.

\subsection{1. $\left(R_{\mathrm{a}}\right)-N$-Benzyl- $N-(1,11-$}

dioxa [11]paracyclophan-13-yl)dinaphtho[2,1d:1', $\left.2^{\prime}-f\right][1,3,2]$ dioxaphosphepin-4-amine $\left(\left(R_{\mathrm{a}}\right)-\right.$ L 1)

Foamy white solid. PTLC (eluent: hexane/EtOAc $=5 / 1$ ). Yield: $26.4 \mathrm{mg}, 41 \%$. Mp: $124-128{ }^{\circ} \mathrm{C}$. ${ }^{1} \mathrm{H}$ NMR (ppm) $\delta 8.04-7.90$ (m, $4 \mathrm{H}), 7.68(\mathrm{~d}, J=8.8 \mathrm{~Hz}, 1 \mathrm{H}), 7.57(\mathrm{~d}, J=8.7 \mathrm{~Hz}, 1 \mathrm{H}), 7.45-7.36$ (m, 4H), 7.27-7.24 (m, 3H), 7.06-7.04 (m, 4H), 6.97 (d, $J=8.7$ $\mathrm{Hz}, 1 \mathrm{H}), 6.77(\mathrm{~d}, J=8.1 \mathrm{~Hz}, 1 \mathrm{H}), 6.53(\mathrm{~s}, 1 \mathrm{H}), 4.56-4.51(\mathrm{~m}$, $1 \mathrm{H}), 4.43-4.35$ (m, 2H), 4.23 (dd, $J=14.4,3.4 \mathrm{~Hz}, 1 \mathrm{H}), 3.97-$ $3.88(\mathrm{~m}, 2 \mathrm{H}), 1.82-1.77(\mathrm{~m}, 2 \mathrm{H}), 1.45-1.42(\mathrm{~m}, 2 \mathrm{H}), 0.95-0.80$ $(\mathrm{m}, 8 \mathrm{H}), 0.72-0.71(\mathrm{~m}, 2 \mathrm{H}) ;{ }^{13} \mathrm{C}$ NMR $(\mathrm{ppm}) \delta 154.8,150.0$, $149.2,142.5,139.6,138.5,137.9,134.8,132.8,132.6,131.5$, $130.8,130.3,129.1,128.3,127.9,127.1,127.0,126.8,126.1$, $124.9,124.7,124.2,124.1,122.6,122.2,122.1,122.0,121.6$, $118.3,71.4,50.3,30.1,29.4,27.6,26.5,25.9,25.8,25.3$ (a pair of peak at aliphatic region is overlapped.); ${ }^{31} \mathrm{P}(\mathrm{ppm}) \delta 140.3$. IR $\left(\mathrm{cm}^{-1}\right) 3749,3735,3649,2921,2361,2343,1734,1363,1229$, 420; HRMS(ESI) calcd for $\mathrm{C}_{42} \mathrm{H}_{40} \mathrm{NNaO}_{4} \mathrm{P}[\mathrm{M}+\mathrm{Na}]^{+}$: 676.2584; found: 676.2587. $[\alpha]_{\mathrm{D}}^{23}=-193.7\left(c 0.91, \mathrm{CHCl}_{3}\right)$.

\subsection{2. $\left(S_{\mathrm{a}}\right)-N$-Benzyl- $N-(1,11-$}

dioxa[11]paracyclophan-13-yl)dinaphtho[2,1-

d: $\left.1^{\prime}, 2^{\prime}-f\right][1,3,2]$ dioxaphosphepin-4-amine $\left(\left(S_{a}\right)-\right.$ L1)

Foamy white solid. PTLC (eluent: hexane/EtOAc $=5 / 1$ ). Yield: $41.6 \mathrm{mg}, 65 \%$. Mp: $126-130{ }^{\circ} \mathrm{C} .{ }^{1} \mathrm{H}$ NMR (ppm) $\delta 7.98(\mathrm{~d}, J=$ $8.5 \mathrm{~Hz}, 1 \mathrm{H}), 7.91(\mathrm{~d}, J=7.5 \mathrm{~Hz}, 1 \mathrm{H}), 7.83(\mathrm{~d}, J=6.6 \mathrm{~Hz}, 1 \mathrm{H})$, $7.75(\mathrm{~d}, J=8.6 \mathrm{~Hz}, 1 \mathrm{H}), 7.52(\mathrm{~d}, J=8.1 \mathrm{~Hz}, 1 \mathrm{H}), 7.46-7.33(\mathrm{~m}$, $5 \mathrm{H}), 7.25-7.12(\mathrm{~m}, 7 \mathrm{H}), 6.91(\mathrm{brs}, 2 \mathrm{H}), 6.79(\mathrm{~d}, J=6.9 \mathrm{~Hz}, 1 \mathrm{H})$, $4.62(\mathrm{~d}, J=15.3 \mathrm{~Hz}, 1 \mathrm{H}), 4.39-4.21(\mathrm{~m}, 3 \mathrm{H}), 4.13-4.03(\mathrm{~m}, 2 \mathrm{H})$, $1.76-1.50(\mathrm{~m}, 4 \mathrm{H}), 1.00-0.79(\mathrm{~m}, 10 \mathrm{H}) ;{ }^{13} \mathrm{C} \mathrm{NMR}(\mathrm{ppm}) \delta 155.1$, $149.8,149.8,149.7,149.0,138.3,135.7,135.5,132.8,132.6$, $131.4,130.6,130.4,129.8,128.4,128.3,128.2,127.9,127.1$, $127.0,126.6,126.1,126.0,124.9,124.5,122.0,121.9,121.0$, $120.9,118.1,71.5,70.8,49.8,30.3,29.6,27.7,26.6,26.3,25.1$, $25.3 ;{ }^{31} \mathrm{P}(\mathrm{ppm}) \delta$ 140.7. IR $\left(\mathrm{cm}^{-1}\right) 2924,2854,2361,1601$, 1508, 1457, 1223, 966, 813, 445; HRMS(ESI) calcd for $\mathrm{C}_{42} \mathrm{H}_{40} \mathrm{NNaO}_{4} \mathrm{P}[\mathrm{M}+\mathrm{Na}]^{+}:$676.2584; found: $676.2587 .[\alpha]_{\mathrm{D}}^{23}=-$ $61.9\left(c 0.51, \mathrm{CHCl}_{3}\right)$.

4.4.3. $\left(\boldsymbol{R}_{\mathrm{a}}\right)-N$-Benzyl- $N-(1,11-$ dioxa [11] paracyclophan-13ylmethyl)dinaphtho[2,1-d:1 ', 2'f] $[1,3,2]$ dioxaphosphepin-4-amine $\left(\left(R_{\mathrm{a}}\right)-\mathrm{L} 2\right)$

Foamy white solid. PTLC (eluent: hexane/EtOAc $=3 / 1$ ). Yield: $25.2 \mathrm{mg}, 36 \%$. Mp: $113-117{ }^{\circ} \mathrm{C}$. ${ }^{1} \mathrm{H}$ NMR (ppm) $\delta 7.98$ (d, $J=$ 
$8.8 \mathrm{~Hz}, 1 \mathrm{H}), 7.91(\mathrm{~d}, J=8.1 \mathrm{~Hz}, 1 \mathrm{H}), 7.82(\mathrm{~d}, J=8.1 \mathrm{~Hz}, 1 \mathrm{H})$, $7.76(\mathrm{~d}, J=8.8 \mathrm{~Hz}, 1 \mathrm{H}), 7.61(\mathrm{~d}, J=8.8 \mathrm{~Hz}, 1 \mathrm{H}), 7.39(\mathrm{dd}, J=$ 7.7, 7.7 Hz, 1H), 7.35-7.30 (m, 3H), $7.28(\mathrm{~d}, J=7.5 \mathrm{~Hz}, 2 \mathrm{H})$, 7.25-7.18 (m, 5H), $7.15(\mathrm{~d}, J=8.7 \mathrm{~Hz}, 1 \mathrm{H}), 7.10-7.03(\mathrm{~m}, 2 \mathrm{H})$, 6.92 (brs, $1 \mathrm{H}), 4.51(\mathrm{dd}, J=15.3,10.3 \mathrm{~Hz}, 1 \mathrm{H}), 4.20-4.08(\mathrm{~m}$, $4 \mathrm{H}), 4.05$ (dd, $J=15.3,4.4 \mathrm{~Hz}, 1 \mathrm{H}), 3.69$ (dd, $J=18.7,15.4 \mathrm{~Hz}$, $1 \mathrm{H}), 3.38$ (dd, $J=15.3,5.9 \mathrm{~Hz}, 1 \mathrm{H}), 1.72-1.46(\mathrm{~m}, 4 \mathrm{H}), 0.93-$ $0.68(\mathrm{~m}, 10 \mathrm{H}) ;{ }^{13} \mathrm{C}$ NMR (ppm) $\delta 154.0,153.3,149.8,149.5$, $138.0,132.8,132.5,131.4,130.7,130.7,130.1,130.0,128.4$, $128.3,128.2,127.0,127.0,126.9,126.0,125.9,124.7,124.5$, $124.1,124.1,122.7,122.4,122.2,121.9,120.3,120.1,72.0$, $71.0,47.3,44.8,30.0,29.6,27.8,26.2,26.1,26.0,25.2 ;{ }^{31} \mathrm{P}$ (ppm) $\delta$ 147.0. IR $\left(\mathrm{cm}^{-1}\right) 2924,2852,2361,2329,1490,1457$, 1230, 1200, 936, 822, 420; HRMS(ESI) calcd for $\mathrm{C}_{43} \mathrm{H}_{42} \mathrm{NNaO}_{4} \mathrm{P}[\mathrm{M}+\mathrm{Na}]^{+}:$690.2739; found: 690.2744 . [ $\left.\alpha\right]_{\mathrm{D}}^{26}=-$ $113.0\left(\right.$ ( $\left.1.06, \mathrm{CHCl}_{3}\right)$.

\subsection{4. $\left(S_{\mathrm{a}}\right)-N$-Benzyl- $N-(1,11-$ dioxa[11]paracyclophan-13- ylmethyl)dinaphtho[2,1-d: $1^{\prime}, 2^{\prime}-$ f] $[1,3,2]$ dioxaphosphepin-4-amine $\left(\left(S_{\mathrm{a}}\right)-\mathrm{L} 2\right)$}

Foamy white solid. PTLC (eluent: hexane/EtOAc $=3 / 1$ ). Yield: $32.8 \mathrm{mg}, 45 \%$. Mp: $114-118{ }^{\circ} \mathrm{C} .{ }^{1} \mathrm{H}$ NMR (ppm) $\delta 7.98$ (dd, $J=$ $8.8,3.1 \mathrm{~Hz}, 1 \mathrm{H}), 7.93$ (dd, $J=8.3,4.2 \mathrm{~Hz}, 1 \mathrm{H}), 7.84-7.81(\mathrm{~m}$, $2 \mathrm{H}), 7.56-7.53(\mathrm{~m}, 1 \mathrm{H}), 7.44-7.31(\mathrm{~m}, 8 \mathrm{H}), 7.27-7.26(\mathrm{~m}, 2 \mathrm{H})$, 7.21-7.13 (m, 3H), 6.86-6.77 (m, 2H), 4.62-4.55 (m, 1H), 4.35$4.28(\mathrm{~m}, 1 \mathrm{H}), 4.20-4.12(\mathrm{~m}, 1 \mathrm{H}), 3.83-3.67(\mathrm{~m}, 4 \mathrm{H}), 3.50-3.44$ $(\mathrm{m}, 1 \mathrm{H}), 1.65-1.56(\mathrm{~m}, 3 \mathrm{H}), 0.82-0.12(\mathrm{~m}, 11 \mathrm{H}) ;{ }^{13} \mathrm{C} \mathrm{NMR}$ (ppm) $\delta 155.0,152.3,149.8,149.7,149.2,138.3,132.8,132.5$, $131.3,130.8,130.7,130.2,130.1,128.9,128.4,128.2,128.1$, $127.5,127.0,126.9,126.1,125.9,124.8,124.5,122.5,122.0$, $121.6,120.5,119.6,119.2,71.5,71.3,50.6,42.5,30.2,29.3$, $27.3,25.4,25.2,24.5$ (a pair of peak at aliphatic region is overlapped); ${ }^{31} \mathrm{P}$ (ppm) $\delta$ 148.7. IR $\left(\mathrm{cm}^{-1}\right)$ 2929, 2362, 2343, 2328, 1507, 1489, 1230, 421; HRMS(ESI) calcd for $\mathrm{C}_{43} \mathrm{H}_{42} \mathrm{NNaO}_{4} \mathrm{P}[\mathrm{M}+\mathrm{Na}]^{+}: 690.2740$; found: 690.2744 . $[\alpha]_{\mathrm{D}}^{24}=$ $+174.5\left(c 1.10, \mathrm{CHCl}_{3}\right)$.

4.4.5. $\left(R_{\mathrm{a}}\right)-N-(1,11-\mathrm{Diox}$ a $[11]$ paracycloph a $-13-$ yl)- $N$-methyldinaphtho $\left[2,1-d: 1^{\prime}, 2^{\prime}\right.$ -

f] $[1,3,2]$ dioxaphosphepin-4-amine $\left(\left(R_{a}\right)-L 3\right)$

Foamy white solid. PTLC (eluent: toluene only). Yield: $17.1 \mathrm{mg}$, 30\%. Mp: $110-115{ }^{\circ} \mathrm{C}$. ${ }^{1} \mathrm{H}$ NMR (ppm) $\delta 7.99$ (dd, $J=8.8,8.8$ $\mathrm{Hz}, 2 \mathrm{H}), 7.94$ (dd, $J=8.1,3.3 \mathrm{~Hz}, 2 \mathrm{H}), 7.59$ (dd, $J=12.0,8.8$ $\mathrm{Hz}, 2 \mathrm{H}), 7.46-7.41$ (m, 3H), 7.35 (d, $J=8.6 \mathrm{~Hz}, 1 \mathrm{H}), 7.31-7.29$ $(\mathrm{m}, 1 \mathrm{H}), 7.27(\mathrm{~s}, 1 \mathrm{H}), 7.07(\mathrm{~d}, J=7.8 \mathrm{~Hz}, 1 \mathrm{H}), 6.90-6.87(\mathrm{~m}$, $2 \mathrm{H}), 4.46-4.41(\mathrm{~m}, 1 \mathrm{H}), 4.34-4.30(\mathrm{~m}, 1 \mathrm{H}), 4.18-4.14(\mathrm{~m}, 2 \mathrm{H})$, $2.73(\mathrm{~s}, 3 \mathrm{H}), 1.78-1.77(\mathrm{~m}, 1 \mathrm{H}), 1.71-1.65(\mathrm{~m}, 2 \mathrm{H}), 1.05-0.84$ (m, 11H); ${ }^{13} \mathrm{C}$ NMR (ppm) $\delta 155.7,149.3,149.1,132.8,132.6$, $131.5,130.7,130.4,130.1,128.3,128.3,127.0,126.1,124.9$, $124.7,124.2,124.0,124.0,122.7,122.2,119.1,119.0,117.8$, 117.6, 71.6, 71.4, 34.9, 30.3, 29.6, 27.7, 26.3, 26.1, 26.0, 25.3 (two pairs of peaks in the aromatic region are overlapped); ${ }^{31} \mathrm{P}$ (ppm) $\delta$ 144.0. IR $\left(\mathrm{cm}^{-1}\right)$ 2924, 2362, 2328, 1746, 1734, 1539, 1507, 1456, 1229, 420; HRMS(ESI) calcd for $\mathrm{C}_{36} \mathrm{H}_{36} \mathrm{NNaO}_{4} \mathrm{P}$ $[\mathrm{M}+\mathrm{Na}]^{+}: 600.2274$; found: $600.2274 .[\alpha]_{\mathrm{D}}^{28}=-124.7(c \quad 0.43$, $\left.\mathrm{CHCl}_{3}\right)$.

4.4.6. $\left(R_{\mathrm{a}}\right)-N-(1,11-\mathrm{Dioxa}[11]$ paracyclophan-13yl)- $N$-is op ropyldin aph tho $\left[2,1-d: 1^{\prime}, 2^{\prime}-\right.$ f] $[1,3,2]$ dioxaphosphepin-4-amine $\left(\left(R_{a}\right)-L 4\right)$

Foamy white solid. PTLC (eluent: hexane/EtOAc $=5 / 1)$. Yield: $12.4 \mathrm{mg}, 20 \%$. Mp: $116-120{ }^{\circ} \mathrm{C} .{ }^{1} \mathrm{H}$ NMR (ppm) $\delta 8.0-7.89(\mathrm{~m}$, 4H), $7.67(\mathrm{~d}, J=8.7 \mathrm{~Hz}, 1 \mathrm{H}), 7.56(\mathrm{~d}, J=8.7 \mathrm{~Hz}, 1 \mathrm{H}), 7.42-7.35$ (m, 4H), 7.26-7.24 (m, 2H), $7.05(\mathrm{~d}, J=8.8 \mathrm{~Hz}, 1 \mathrm{H}), 6.95(\mathrm{~d}, J$ $=8.1 \mathrm{~Hz}, 1 \mathrm{H}), 6.65(\mathrm{~s}, 1 \mathrm{H}), 4.50-4.37(\mathrm{~m}, 2 \mathrm{H}), 3.99(\mathrm{~d}, J=8.4$ $\mathrm{Hz}, 1 \mathrm{H}), 3.78(\mathrm{~s}, 1 \mathrm{H}), 3.58-3.53(\mathrm{~m}, 1 \mathrm{H}), 1.87-1.83(\mathrm{~m}, 1 \mathrm{H})$, $1.72-1.66(\mathrm{~m}, 1 \mathrm{H}), 1.42-1.20(\mathrm{~m}, 2 \mathrm{H}), 0.99-0.85(\mathrm{~m}, 8 \mathrm{H}), 0.96$ $(\mathrm{d}, J=6.3 \mathrm{~Hz}, 3 \mathrm{H}), 0.88(\mathrm{~d}, J=6.4 \mathrm{~Hz}, 3 \mathrm{H}), 0.80-0.66(\mathrm{~m}, 2 \mathrm{H})$; ${ }^{13} \mathrm{C}$ NMR (ppm) $\delta 179.8,178.8,154.1,151.7,150.1,149.8$, $132.9,132.7,131.4,130.8,130.1,129.8,128.9,128.4,128.3$, $127.7,127.1,127.1,126.0,125.9,124.7,124.4,122.6,122.3$, 120.4, 119.9, 71.7, 71.2, 53.4, 30.2, 29.7, 29.6, 27.7, 26.6, 26.0, $25.6,22.9,21.5 ;{ }^{31} \mathrm{P}(\mathrm{ppm}) \delta 144.4$. IR $\left(\mathrm{cm}^{-1}\right) 2926,2360,2339$, $1735,1540,1509,1490,1363,1231,948,517,458,420$; HRMS(ESI) calcd for $\mathrm{C}_{38} \mathrm{H}_{40} \mathrm{NNaO}_{4} \mathrm{P}[\mathrm{M}+\mathrm{Na}]^{+}$: 628.2584; found: $628.2587 .[\alpha]_{\mathrm{D}}^{30}=-142.5\left(c 0.87, \mathrm{CHCl}_{3}\right)$.

4.4.7. $N$-Benzyl$N-(1,11-$ dioxa[11]paracyclophan-13yl)dibenzo[d,f $][1,3,2]$ dioxaphosphepin-6-amine (L5)

Foamy white solid. PTLC (eluent: toluene only). Yield: $38.9 \mathrm{mg}$, 70\%. Mp: $68-74{ }^{\circ} \mathrm{C} .{ }^{1} \mathrm{H}$ NMR (ppm) $\delta 7.48(\mathrm{~m}, 2 \mathrm{H}), 7.36-7.26$ $(\mathrm{m}, 6 \mathrm{H}), 7.11-7.05(\mathrm{~m}, 5 \mathrm{H}), 6.97-6.93(\mathrm{~m}, 1 \mathrm{H}), 6.78-6.72(\mathrm{~m}$, $2 \mathrm{H}), 4.61-4.35(\mathrm{~m}, 4 \mathrm{H}), 4.12-4.06(\mathrm{~m}, 2 \mathrm{H}), 1.72-1.61(\mathrm{~m}, 3 \mathrm{H})$, $0.92-0.78(\mathrm{~m}, 11 \mathrm{H}) ;{ }^{13} \mathrm{C}$ NMR $(\mathrm{ppm}) \delta 155.1,151.9,150.9$, $149.9,138.2,135.3,135.1,131.3,130.6,129.9,129.8,129.5$, $129.3,129.0,128.0,126.8,125.0,124.6,122.5,122.2,122.1$, $121.5,121.4,118.1,71.6,71.3,49.8,30.4,29.6,27.8,26.5,26.1$, $25.9,25.4 ;{ }^{31} \mathrm{P}(\mathrm{ppm}) \delta 141.0$. IR $\left(\mathrm{cm}^{-1}\right) 2924,2852,2361,1493$, 1474, 1457, 1436, 1246, 1192, 1166, 885; HRMS(ESI) calcd for $\mathrm{C}_{34} \mathrm{H}_{36} \mathrm{O}_{4} \mathrm{NNaP}[\mathrm{M}+\mathrm{Na}]^{+}: 576.2280$; found: $576.2274 .[\alpha]_{\mathrm{D}}^{24}=-$ 90.5 (c $\left.0.53, \mathrm{CHCl}_{3}\right)$.

\subsection{8. $N$-Benzyl-}

dioxa[11]paracyclophan-13-

ylmethyl)dibenzo[d,f $][1,3,2]$ dioxaphosphepin-6amine (L6)

Foamy white solid. PTLC (eluent: hexane/EtOAc $=5 / 1$ ). Yield: $26.5 \mathrm{mg}, 47 \%$. Mp: $65-70{ }^{\circ} \mathrm{C} .{ }^{1} \mathrm{H}$ NMR (ppm) $\delta 7.42(\mathrm{~d}, J$ $=7.5 \mathrm{~Hz}, 1 \mathrm{H}), 7.39-7.36(\mathrm{~m}, 5 \mathrm{H}), 7.34-7.24(\mathrm{~m}, 4 \mathrm{H}), 7.22-7.16$ $(\mathrm{m}, 3 \mathrm{H}), 7.04(\mathrm{~d}, J=7.9 \mathrm{~Hz}, 1 \mathrm{H}), 6.91-6.90(\mathrm{~m}, 2 \mathrm{H}), 4.41-4.35$ (m, 1H), 4.29-4.24 (m, 1H), 4.20-4.15 (m, 1H), 4.13-3.93 (m, $4 \mathrm{H}), 3.89-3.86(\mathrm{~m}, 1 \mathrm{H}), 1.71-1.54(\mathrm{~m}, 3 \mathrm{H}), 1.16-1.09(\mathrm{~m}, 1 \mathrm{H})$, $0.88-0.75(\mathrm{~m}, 3 \mathrm{H}), 0.56-0.51(\mathrm{~m}, 7 \mathrm{H}) ;{ }^{13} \mathrm{C} \mathrm{NMR}(\mathrm{ppm}) \delta 154.8$, $152.5,151.9,151.8,138.3,131.4,131.4,130.4,130.4,129.7$, $129.5,129.3,129.0,128.6,128.3,127.2,124.7,124.2,122.2$, $121.7,120.2,120.1,119.5,71.8,71.1,49.4,43.3,30.1,29.7$, $27.6,25.8,25.6,25.6,25.0$ (a pair of peaks in the aromatic region is overlapped); ${ }^{31} \mathrm{P}(\mathrm{ppm}) \delta 149.1$. IR $\left(\mathrm{cm}^{-1}\right) 2927,2361$, 2341, 1734, 1541, 1489, 1436, 1191, 884, 772, 447, 419; HRMS(ESI) calcd for $\mathrm{C}_{35} \mathrm{H}_{38} \mathrm{NNaO}_{4} \mathrm{P} \quad[\mathrm{M}+\mathrm{Na}]^{+}$: 590.2430; found: $590.2431 .[\alpha]_{\mathrm{D}}^{20}=-8.7\left(c 0.61, \mathrm{CHCl}_{3}\right)$.

\subsection{9. $N-(1,11-D i o x a[11]$ paracyclophan-13- ylmethyl)- $N$ - \\ isopropyldibenzo[d,f] $[1,3,2]$ dioxaphosphepin-6- amine (L 7)}

Foamy white solid. PTLC (eluent: hexane/EtOAc $=5 / 1$ ). Yield: $15.4 \mathrm{mg}, 30 \%$. Mp: $130-134{ }^{\circ} \mathrm{C}$. ${ }^{1} \mathrm{H}$ NMR (ppm) $\delta 7.42(\mathrm{dd}, J=$ $7.6,1.6 \mathrm{~Hz}, 1 \mathrm{H}), 7.38(\mathrm{dd}, J=7.6,1.6 \mathrm{~Hz}, 1 \mathrm{H}), 7.35(\mathrm{dd}, J=$ $7.7,1.8 \mathrm{~Hz}, 1 \mathrm{H}), 7.31(\mathrm{dd}, J=7.7,1.6 \mathrm{~Hz}, 1 \mathrm{H}), 7.23-7.17(\mathrm{~m}$, $5 \mathrm{H}), 6.80(\mathrm{~d}, J=1.6 \mathrm{~Hz}, 2 \mathrm{H}), 4.26-4.19(\mathrm{~m}, 1 \mathrm{H}), 4.17-4.13(\mathrm{~m}$, $2 \mathrm{H}), 4.04-3.97(\mathrm{~m}, 2 \mathrm{H}), 3.85(\mathrm{ddd}, J=12.0,6.6,3.1 \mathrm{~Hz}, 1 \mathrm{H})$, 3.69-3.61 (m, 1H), 1.65-1.58 (m, 1H), 1.56-1.48 (m, 1H), 1.43 $(\mathrm{d}, J=6.7 \mathrm{~Hz}, 3 \mathrm{H}), 1.27-1.23(\mathrm{~m}, 1 \mathrm{H}), 1.14(\mathrm{~d}, J=6.7 \mathrm{~Hz}, 3 \mathrm{H})$, $1.10-1.05(\mathrm{~m}, 1 \mathrm{H}), 0.81-0.74(\mathrm{~m}, 2 \mathrm{H}), 0.52-0.39(\mathrm{~m}, 8 \mathrm{H}) ;{ }^{13} \mathrm{C}$ NMR (ppm) $\delta 154.8,152.6,152.6,151.4,133.5,130.3,130.3$, $129.9,129.5,129.3,128.9,124.7,124.0,122.4,121.7,120.2$, $119.7,118.9,71.7,71.2,49.2,41.3,30.2,29.7,27.5,25.8,25.6$, 25.5, 25.0, 23.2, 23.0; ${ }^{31} \mathrm{P}$ (ppm) $\delta 151.3$. IR $\left(\mathrm{cm}^{-1}\right) 2924,2852$, 2362, 1488, 1435, 1247, 1191, 1095, 1036, 888, 847, 765, 419; 
HRMS(ESI) calcd for $\mathrm{C}_{31} \mathrm{H}_{38} \mathrm{NNaO}_{4} \mathrm{P} \quad[\mathrm{M}+\mathrm{Na}]^{+}$: 542.2432; found: $542.2431 .[\alpha]_{\mathrm{D}}^{28}=+86.9\left(c 0.84, \mathrm{CHCl}_{3}\right)$.

4.4.10. $N$-Benzyl- $N-(1,11-$

dioxa $[11]$ a a acyclophan-13-yl) $-2,4,8,10$ -

tetramethyldibenzo[d,f] $[1,3,2]$ dioxaphosphepin6-amine (L8)

Foamy white solid. PTLC (eluent: toluene only). Yield: $33.2 \mathrm{mg}$, 55\%. Mp: 82-86 ${ }^{\circ} \mathrm{C} .{ }^{1} \mathrm{H}$ NMR (ppm) $\delta 7.14-7.11$ (m, 3H), 7.09$7.05(\mathrm{~m}, 6 \mathrm{H}), 6.96(\mathrm{~d}, J=8.6 \mathrm{~Hz}, 1 \mathrm{H}), 6.80-6.77(\mathrm{~m}, 2 \mathrm{H}), 4.67$ $(\mathrm{d}, J=14.4 \mathrm{~Hz}, 1 \mathrm{H}), 4.42(\mathrm{dd}, J=14.4,2.2 \mathrm{~Hz}, 1 \mathrm{H}), 4.24$ (dd, $J$ $=5.1,5.1 \mathrm{~Hz}, 2 \mathrm{H}), 4.20-4.07(\mathrm{~m}, 2 \mathrm{H}), 2.47(\mathrm{~s}, 3 \mathrm{H}), 2.46(\mathrm{~s}, 3 \mathrm{H})$, 2.36 (s, 3H), 2.33 (s, 3H), 1.65-1.58 (m, 2H), 1.39-1.37 (m, 2H), $0.96-0.83(\mathrm{~m}, 4 \mathrm{H}), 0.80-0.67(\mathrm{~m}, 6 \mathrm{H}) ;{ }^{13} \mathrm{C}$ NMR (ppm) $\delta 154.9$, $149.3,149.2,148.1,148.0,146.5,137.8,135.6,135.4,133.8$, $132.8,131.2,131.1,129.8,129.6,128.1,127.8,126.8,122.0$, $120.1,120.0,117.3,71.4,71.2,48.5,30.0,29.1,27.4,26.3,25.9$ 25.6, 25.2, 20.9, 20.8, 17.0, 16.7; ${ }^{31} \mathrm{P}(\mathrm{ppm}) \delta 134.7 . \mathrm{IR}\left(\mathrm{cm}^{-1}\right)$ 2923, 2852, 1600, 1494, 1478, 1457, 1409, 1245, 1213, 1193, 1154, 1120, 1065, 857, 759, 699, 593, 476, 418; HRMS(ESI) calcd for $\mathrm{C}_{38} \mathrm{H}_{44} \mathrm{O}_{4} \mathrm{NNaP}[\mathrm{M}+\mathrm{Na}]^{+}: 632.2897$; found: 632.2900 . $[\alpha]_{\mathrm{D}}^{27}=-123.3\left(c 0.56, \mathrm{CHCl}_{3}\right)$.

4.4.11. $N$-Benzyl- $N-(1,11$ -

dioxa[11]paracyclophan-13-ylmethyl)-2,4,8,10tetramethyldibenzo $[d, f][1,3,2]$ dioxaphosphepin6-amine (L9)

White solid. PTLC (eluent: hexane/EtOAc $=5 / 1$ ). Yield: 24.5 mg, 39\%. Mp: $76-80{ }^{\circ} \mathrm{C} .{ }^{1} \mathrm{H}$ NMR (ppm) $\delta 7.37$ (d, $J=6.8 \mathrm{~Hz}$, $2 \mathrm{H}), 7.31(\mathrm{dd}, J=7.6,7.6 \mathrm{~Hz}, 2 \mathrm{H}), 7.27-7.26(\mathrm{~m}, 1 \mathrm{H}), 7.14(\mathrm{~s}$, $1 \mathrm{H}), 7.01-6.98(\mathrm{~m}, 3 \mathrm{H}), 6.92-6.90(\mathrm{~m}, 3 \mathrm{H}), 4.24-4.20(\mathrm{~m}, 1 \mathrm{H})$, 4.15-3.97 (m, 5H), $3.92(\mathrm{~d}, J=14.7, \mathrm{~Hz}, 2 \mathrm{H}), 2.36(\mathrm{~s}, 3 \mathrm{H}), 2.32$ (s, 3H), 2.27 (s, 3H), 2.04 (s, 3H), 1.29-1.27 (m, 3H), 0.82-0.52 $(\mathrm{m}, 11 \mathrm{H}) ;{ }^{13} \mathrm{C}$ NMR $(\mathrm{ppm}) \delta 154.8,152.7,149.6,147.9,146.7$, $138.1,133.2,132.6,131.3,130.9,130.9,130.8,130.0,129.9$, $129.2,128.2,127.8,127.7,127.2,120.3,119.5,99.9,71.6,71.4$, $30.2,29.7,27.7,25.9,25.6,25.5,25.2,20.8,20.8,16.5,16.5$. (two pairs of peaks are overlapped at aliphatic region). ${ }^{31} \mathrm{P}(\mathrm{ppm})$ $\delta$ 143.0. IR $\left(\mathrm{cm}^{-1}\right)$ 2923, 2851, 2362, 2338, 1735, 1489, 1363, 1214, 1194, 854, 420; HRMS(ESI) calcd for $\mathrm{C}_{39} \mathrm{H}_{46} \mathrm{NNaO}_{4} \mathrm{P}$ $[\mathrm{M}+\mathrm{Na}]^{+}:$646.3054; found: 646.3057. $[\alpha]_{\mathrm{D}}^{25}=+24.1$ (c 1.27, $\left.\mathrm{CHCl}_{3}\right)$.

4.4.12. $\quad N$-(2,5-dimethoxyphenyl)- $N$-methyldinaphtho[2,1d:1',2'-f] [1,3,2]dioxaphosphepin-4-amine (L10)

Foamy white solid. PTLC (eluent: toluene only). Yield: $13.9 \mathrm{mg}$, 29\%. Mp: 89-92 ${ }^{\circ} \mathrm{C} .{ }^{1} \mathrm{H}$ NMR (ppm) $\delta$ 7.97-7.89 (m, 4H), 7.56$7.53(\mathrm{~m}, 2 \mathrm{H}), 7.45-7.33(\mathrm{~m}, 4 \mathrm{H}), 7.28-7.22(\mathrm{~m}, 2 \mathrm{H}), 6.88-6.84$ $(\mathrm{m}, 2 \mathrm{H}), 6.72-6.69(\mathrm{~m}, 1 \mathrm{H}), 3.89-3.87(\mathrm{~m}, 3 \mathrm{H}), 3.75-3.73(\mathrm{~m}$, $3 \mathrm{H}), 2.59-2.57(\mathrm{~m}, 3 \mathrm{H}) ;{ }^{13} \mathrm{C}$ NMR $(\mathrm{ppm}) \delta 153.9,150.2,150.1$, $149.5,135.2,135.0,132.9,131.4,130.8,130.2,129.9,128.3$, $128.2,127.0,126.9,126.0,124.0,124.0,122.7,122.2,122.0$, $115.0,114.9,113.4,111.1,99.9,56.6,55.7,35.0 ;{ }^{31} \mathrm{P}(\mathrm{ppm}) \delta$ 144.7. IR $\left(\mathrm{cm}^{-1}\right) 2927,1614,1587,1500,1460,1327,1269$, 1221，1063，937，820; HRMS(ESI) calcd for $\mathrm{C}_{29} \mathrm{H}_{25} \mathrm{NO}_{4} \mathrm{P}$ $[\mathrm{M}+\mathrm{H}]^{+}:$482.1517; found: 482.1516. $[\alpha]_{\mathrm{D}}^{32 .}=-72.1(c 0.77$, $\left.\mathrm{CHCl}_{3}\right)$.

\section{Acknowledgments}

This work was supported by JST, ACT-C, Japan. We are grateful to Tokyo Chemical Industry Co., Ltd. (TCI) for generous supports in 1,11-dioxa[11]paracyclophane supply.

\section{References and notes}

1. Ojima, I. Catalytic Asymmetric Synthesis, Wiley, New York, 2000.

2. Blaser, H. U.; Schmidt, E. Asymmetric Catalysis on Industrial Scale, Wiley, New York, 2004.

3. Noyori, R. Asymmetric Catalysis in Organic Synthesis, Wiley, New York, 1994.

4. For few important examples see (a) Tang, W.; Zhang, X. Chem. Rev. 2003, 103, 3029-3069; (b) Alexakis, A.; Benhaim, C. Eur. J. Org. Chem. 2002, 3221-3236; (c) Ansell, J.; Wills, M. Chem. Soc. Rev. 2002, 31, 259-268.

5. Hargaden, G. C.; Guiry, P. J. Chem. Rev. 2009, 109, 25052550.

6. (a) Bravo, M. J.; Ceder, R. M.; Grabulosa, A.; Muller, G.; Rocamora, M.; Bayon, J. C.; Pera, D. Organometallics 2015, 34, 3799-3808; (b) Harada, H.; Thalji, R. K.; Bergman, R. G.; Ellman, J. A. J. Org. Chem. 2008, 73, 6772-6779; (c) Reetz, M. T.; Sell, T. Tetrahedron Lett. 2000, 41, 63336336.

7. Valentine Jr, D.; Johnson, K. K.; Priester, W.; Sun, R. C.; Toth, K.; Saucy, G. J. Org. Chem. 1980, 45, 3698-3703.

8 (a) Lubell, W. D.; Kitamura, M.; Noyori, R. Tetrahedron: Asymmetry 1991, 2, 543-554; (b) Zhou, Y.-G.; Tang, W.; Wang, W.-B.; Li, W.; Zhang, X. J. Am. Chem. Soc. 2002, 124, 4952-4953; (c) Vineyard, B. D.; Knowles, W. S.; Sabacky, M. J.; Bachman, G. L.; Weinkauff, D. J. J. Am. Chem. Soc. 1977, 99, 5946-5952; (d) Knowles, W. S. Angew. Chem. Int. Ed. 2002, 41, 1998-2007; (e) Zhang, Z.; Quian, H.; Longmire, J.; Zhang, X. J. Org. Chem. 2000, 65, 6223-6226.

9 (a) Berg, M.; Minnard, A. J.; Schudde, E. P.; Esch, J.; de Vries, A. H. M.; de Vries, J. G.; Feringa, B. L. J. Am. Chem. Soc. 2002, 122, 11539-11540; (b) Duursma, A.; Boiteau, J.G.; Lefort, L.; Boogers, J. A. F.; de Vries, A. H. M.; de Vries, J. G.; Minnard, A. J.; Feringa, B. L. J. Org. Chem. 2004, 69, 8045-8052; (c) Clever, C.; Fernandez, E.; Gillon, A.; Heslop, K.; Hyett, D. J.; Martorell, A.; Orpen, A. G.; Pringle, P. G. Chem. Commun. 2000, 961-962; (d) Reetz, M. T.; Mehler, G. Angew. Chem. Int. Ed. 2000, 39, 3889-3890; (e) Jia, X.; Li, X.; Xu, L.; Shi, Q.; Yao, X.; Chan, A. S. C. J. Org. Chem. 2003, 68, 4539-4541.

10. Szulc, I.; Kolodzuick, R.; Kryczka, B.; Zawisza, A. Tetrahedron Lett. 2015, 56, 4740-4743.

11. (a) Knowles, W. S.; Sabacky, M. J.; Vineyard, B. D. Chem. Commun. 1972, 10-11; (b) Lega, M.; Margalef, J.; Ruffo, F.; Pamies, O.; Dieguez, M. Tetrahedron: Asymmetry 2013, 24, 995-1000.

12. Boiteau, J.-G.; Imbos, R.; Minnarrd, A. J.; Feringa, B. L. Org. Lett. 2003, 5, 681-684.

13. (a) Teichert, J. F.; Feringa, B. L. Angew. Chem. Int. Ed. 2010, 49, 2486-2528; (b) Liu, Z.; Cao, Z.; Du, H. Org. Biomol. Chem. 2011, 9, 5369-5372; (c) Boeda, F.; Rix, D.; Clavier, H.; Crevisy, C.; Mauduit, M. Tetrahedron: Asymmetry 2006, 17, 2726-2729; (d) Esquivias, J.; Arrayas, R. G.; Carretero, J. C. J. Org. Chem. 2005, 70, 7451-7454.

14. (a) Scafato, P.; Caprioli, F.; Rosini, C. Tetrahedron: Asymmetry 2011, 22, 558-561; (b) Scafato, P.; Cunsolo, G.; Labano, S.; Rosini, C. Tetrahedron 2004, 60, 8801-8806; (c) Trost, B. M.; Donckele, E. J.; Thaisrivongs, D. A.; Osipov, M.; Masters, J. T. J. Am. Chem. Soc. 2015, 137, 2776-2784; (d) Pena, D.; Minaard, A. J.; de Vries, J. G.; Feringa, B. L. J. Am. Chem. Soc. 2002, 124, 14552-14553; (e) How, R. C.; Hembre, R.; Ponasik, J. A.; Tolleson, G. S.; Clarke, M. L. Catal. Sci. Technol. 2016, 6, 118-124; (f) Yang, Z.; Zhou, J. J. Am. Chem. Soc. 2012, 134, 11833-11835. 
15. (a) van der Molen, N. C.; Wegman, T. D. T.; Mastral, M. F.; Feringa, B. L. J. Org. Chem. 2015, 80, 4981-4981; (b) Lin, C.-F.; Chien, C.-W.; Ojima, I. Org. Chem. Front. 2014, 1, 1062-1066.

16. (a) Lee, A.; Ahn, S.; Kang, K.; Seo, M.- S.; Kim, Y.; Kim, W. Y.; Kim, H. Org. Lett. 2014, 16, 5490-5493; (b) Zhao, W.; Wang, T.; Zhao, R.; Xie, H.; Liu, L. Tetrahedron: Asymmetry 2016, 27, 157-162.

17. (a) Kanda, K.; Endo, K.; Shibata, T. Org. Lett. 2010, 12, 1980-1983; (b) Kanda, K.; Hamanaka, R.; Endo, K.; Shibata, T. Tetrahedron 2012, 68, 1407-1416.

18. Brown, C. J.; Farthing, A. C. Nature 1949, 164, 915-916.

19. Han, L.; Lei, Y.; Xing, P.; Zhao, X.-L.; Jiang, B. J. Org. Chem. 2015, 80, 3752-3757.

20. Kanda, K.; Oshima, S.; Shizuno, T.; Hamanaka, R.; Fukai, M.; Shibata, T. Heterocycles 2014, 88, 1355-1370.

21. Shibata, T.; Fukai, M.; Sekine, R.; Hazra, M.; Kanyiva, K. S. Synthesis DOI: 10.1055/s-0035-1562097.

22. Gonzalez, A. Z.; Benitez, D.; Tkatchouk, E.; Goddard, III, W. A.; Toste, F. D. J. Am. Chem. Soc. 2011, 133, 5500 5507.

23. (a) Klimczyk, S.; Misale, A.; Huang, X.; Maulide, N. Angew. Chem. Int. Ed. 2015, 54, 10365-10369; (b) Teller, H.; Flugge, S.; Goddard, R.; Furstner, A. Angew. Chem. Int. Ed. 2010, 49, 1949-1953.

24. Nishiyama, K.; Tanaka, N. J. Chem. Soc., Chem Commun. 1983, 1322-1323.

25. Gavrilov, K. N.; Shiryaev, A. A.; Chuchelkin, I. V.; Zheglov, S. V.; Rastorguev, E. A.; Davankov, V. A.; Borner, A. Tetrahedron: Asymmetry 2012, 23, 1052-1057.

26. (a) Yu, H.; Xie, F.; Ma, Z.; Liu, Y.; Zhang, W. Org. Biomol. Chem. 2012, 10, 5137-5142; (b) Alexakis, A.; Benhaim, C.; Rosset, S.; Humam, M. J. Am. Chem. Soc. 2002, 124, 52625263.

27. The reaction using $\left(R_{\mathrm{a}}\right)-\mathbf{L} \mathbf{3}$ as a chiral ligand was conducted at a lower temperature $\left(-20{ }^{\circ} \mathrm{C}\right)$, but the enantioselectivity was decreased $(93 \%, 53 \%$ ee $(R))$. 


\section{Stereochemistry Abstract}

To create your abstract, type over the instructions in the template box below.

Fonts or abstract dimensions should not be changed or altered. You may insert more abstracts by copying this box or by using the menu option to insert a stereochemistry abstract.

Madhurima Hazra, Kyalo Stephen Kanyiva, and Takanori

Shibata

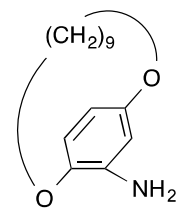

\section{$\mathrm{Ee}=98 \%$}

$[\alpha]_{\mathrm{D}}^{23}=+38.6^{\circ}\left(\right.$ c $0.57, \mathrm{CHCl}_{3}, 98 \%$ ee from HPLC)

Source of chirality: $(+)$-sparteine

$\mathrm{C}_{15} \mathrm{H}_{23} \mathrm{NO}_{2}$

13-Amino-1,11-dioxa[11]paracyclophane

Madhurima Hazra, Kyalo Stephen Kanyiva, and Takanori

Shibata

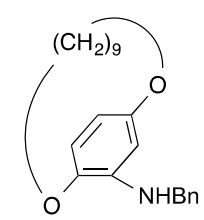

$\mathrm{Ee}=98 \%$

$[\alpha]_{\mathrm{D}}^{25}=+63.2^{\circ}$ (c $0.76, \mathrm{CHCl}_{3}, 98 \%$ ee from HPLC)

Source of chirality: $(+)$-sparteine

$\mathrm{C}_{22} \mathrm{H}_{29} \mathrm{NO}_{2}$

13-Benzylamino-1,11-dioxa[11]paracyclophane

Madhurima Hazra, Kyalo Stephen Kanyiva, and Takanori Shibata

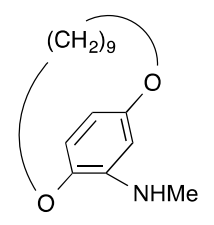

$[\alpha]_{\mathrm{D}}^{26}=+88.2^{\circ}\left(\mathrm{c} 1.32, \mathrm{CHCl}_{3}\right)$

Source of chirality: $(+)$-sparteine

$\mathrm{C}_{16} \mathrm{H}_{2} \mathrm{NO}_{26}$

13-Methylamino-1,11-dioxa[11]paracyclophane 
Madhurima Hazra, Kyalo Stephen Kanyiva, and Takanori

Shibata

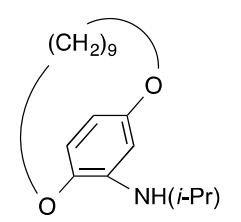

$[\alpha]_{\mathrm{D}}^{25}=+98.4^{\circ}\left(\right.$ c $\left.0.57, \mathrm{CHCl}_{3}\right)$

Source of chirality: $(+)$-sparteine

$\mathrm{C}_{18} \mathrm{H}_{29} \mathrm{NO}_{2}$

13-Isopropylamino-1,11-dioxa[11]paracyclophane

Madhurima Hazra, Kyalo Stephen Kanyiva, and Takanori

Shibata

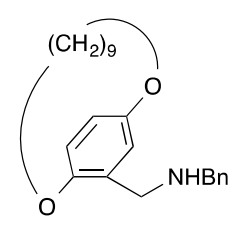

$\mathrm{Ee}=98 \%$

$[\alpha]_{\mathrm{D}}^{26}=+13.0^{\circ}\left(c=0.85, \mathrm{CHCl}_{3}, 98 \%\right.$ ee from HPLC)

Source of chirality: $(+)$-sparteine

$\mathrm{C}_{23} \mathrm{H}_{31} \mathrm{NO}_{2}$

13-(Benzylamino)methyl-1,11-dioxa[11]paracyclophane

Madhurima Hazra, Kyalo Stephen Kanyiva, and Takanori

Shibata

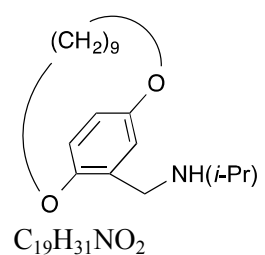

$[\alpha]_{\mathrm{D}}^{28}=+8.8^{\circ}\left(c=0.61, \mathrm{CHCl}_{3}\right)$

Source of chirality: $(+)$-sparteine

$\mathrm{C}_{19} \mathrm{H}_{31} \mathrm{NO}_{2}$

13-(Isopropylamino)methyl-1,11-dioxa[11]paracyclophane

Madhurima Hazra, Kyalo Stephen Kanyiva, and Takanori Shibata

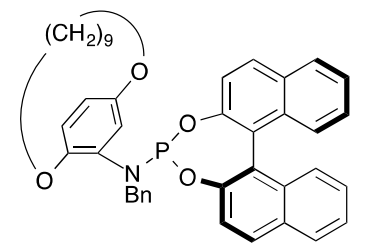

$[\alpha]_{\mathrm{D}}^{23}=-193.7^{\circ}\left(c\right.$ 0.91, $\left.\mathrm{CHCl}_{3}\right)$

Source of planar chirality: $(+)$-sparteine

Source of axial chirality: $(R)$-BINOL

$\mathrm{C}_{42} \mathrm{H}_{40} \mathrm{NO}_{4} \mathrm{P}$

$\left(R_{\mathrm{a}}\right)-N$-Benzyl- $N$-(1,11-dioxa[11] paracyclophan-13-yl)dinaphtho[2,1-d:1',2'-f][1,3,2]dioxaphosphepin-4-amine 
Madhurima Hazra, Kyalo Stephen Kanyiva, and Takanori

Shibata

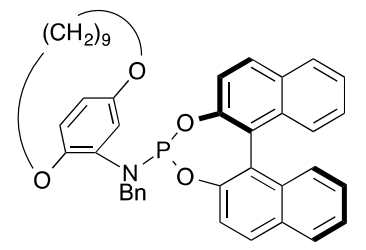

$[\alpha]_{\mathrm{D}}^{23}=-61.9^{\circ}\left(\mathrm{c} 0.51, \mathrm{CHCl}_{3}\right)$

Source of planar chirality: $(+)$-sparteine

Source of axial chirality: (S)-BINOL

$\mathrm{C}_{42} \mathrm{H}_{40} \mathrm{NO}_{4} \mathrm{P}$

$\left(S_{\mathrm{a}}\right)-N$-Benzyl- $N$-(1,11-dioxa[11]paracyclophan-13-yl)dinaphtho[2,1-d:1',2'-f][1,3,2]dioxaphosphepin-4-amine

Madhurima Hazra, Kyalo Stephen Kanyiva, and Takanori

Shibata

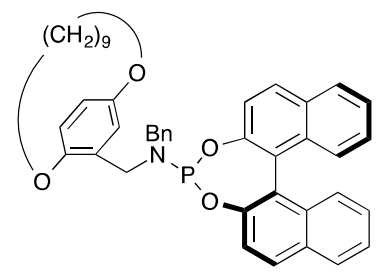

$[\alpha]_{\mathrm{D}}^{26}=-113.0^{\circ}\left(c 1.06, \mathrm{CHCl}_{3}\right)$

Source of planar chirality: $(+)$-sparteine

Source of axial chirality: $(R)-\mathrm{BINOL}$

$\mathrm{C}_{43} \mathrm{H}_{42} \mathrm{NO}_{4} \mathrm{P}$

$\left(R_{\mathrm{a}}\right)-N$-Benzyl- $N$-(1,11-dioxa[11]paracyclophan-13-ylmethyl)dinaphtho[2,1-d:1',2'-f][1,3,2]dioxaphosphepin-4-amine

Madhurima Hazra, Kyalo Stephen Kanyiva, and Takanori

Shibata

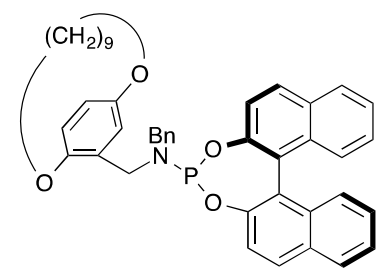

$[\alpha]_{\mathrm{D}}^{24}=+174.5^{\circ}\left(c 1.10, \mathrm{CHCl}_{3}\right)$

Source of planar chirality: (+)-sparteine

Source of axial chirality: (S)-BINOL

$\mathrm{C}_{43} \mathrm{H}_{42} \mathrm{NO}_{4} \mathrm{P}$

$\left(S_{\mathrm{a}}\right)-N$-Benzyl- $N$-(1,11-dioxa[11]paracyclophan-13-ylmethyl)dinaphtho[2,1-d:1',2'-f][1,3,2]dioxaphosphepin-4-amine

Madhurima Hazra, Kyalo Stephen Kanyiva, and Takanori Shibata

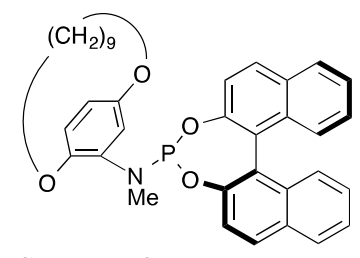

$[\alpha]_{\mathrm{D}}^{28}=-124.7^{\circ}\left(\right.$ c $\left.0.43, \mathrm{CHCl}_{3}\right)$

Source of planar chirality: (+)-sparteine

Source of axial chirality: $(R)$-BINOL

$\mathrm{C}_{36} \mathrm{H}_{36} \mathrm{NNO}_{4} \mathrm{P}$

$\left(R_{\mathrm{a}}\right)-N$-(1,11-Dioxa[11]paracyclophan-13-yl)- $N$-methyldinaphtho[2,1-d:1',2'-f][1,3,2]dioxaphosphepin-4-amine 
Madhurima Hazra, Kyalo Stephen Kanyiva, and Takanori Shibata

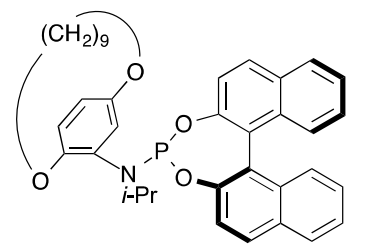

$[\alpha]_{\mathrm{D}}^{30}=-142.5^{\circ}\left(\right.$ c $\left.0.87, \mathrm{CHCl}_{3}\right)$

Source of planar chirality: $(+)$-sparteine

Source of axial chirality: $(R)-\mathrm{BINOL}$

$\mathrm{C}_{38} \mathrm{H}_{40} \mathrm{NO}_{4} \mathrm{P}$

$\left(R_{\mathrm{a}}\right)-N$-(1,11-Dioxa[11]paracyclophan-13-yl)- $N$-isopropyldinaphtho[2,1-d:1',2'-f][1,3,2]dioxaphosphepin-4-amine

Madhurima Hazra, Kyalo Stephen Kanyiva, and Takanori

Shibata

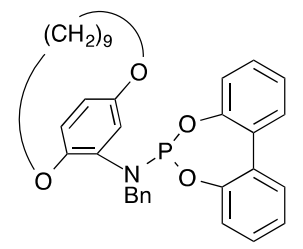

$[\alpha]_{\mathrm{D}}^{24}=-90.5^{\circ}\left(c 0.53, \mathrm{CHCl}_{3}\right)$

Source of planar chirality: $(+)$-sparteine

$\mathrm{C}_{34} \mathrm{H}_{36} \mathrm{NO}_{4} \mathrm{P}$

$N$-Benzyl- $N$-(1,11-dioxa[11]paracyclophan-13-yl)dibenzo[d,f][1,3,2]dioxaphosphepin-6-amine

Madhurima Hazra, Kyalo Stephen Kanyiva, and Takanori

Shibata

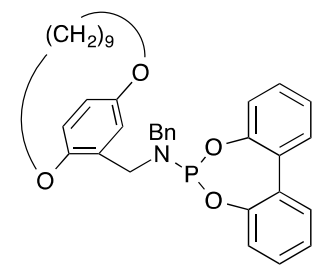

$[\alpha]_{\mathrm{D}}^{20}=-8.7^{\circ}\left(c 0.61, \mathrm{CHCl}_{3}\right)$

Source of planar chirality: $(+)$-sparteine

$\mathrm{C}_{35} \mathrm{H}_{38} \mathrm{NO}_{4} \mathrm{P}$

$N$-Benzyl- $N$-(1,11-dioxa[11]paracyclophan-13-ylmethyl)dibenzo[d,f][1,3,2]dioxaphosphepin-6-amine

Madhurima Hazra, Kyalo Stephen Kanyiva, and Takanori Shibata

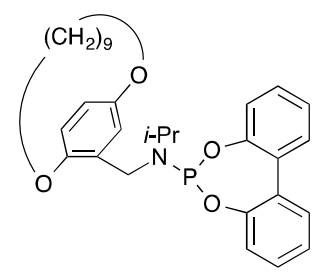

$[\alpha]_{\mathrm{D}}^{28}=+86.9^{\circ}\left(c 0.84, \mathrm{CHCl}_{3}\right)$

Source of planar chirality: $(+)$-sparteine

$\mathrm{C}_{31} \mathrm{H}_{38} \mathrm{NO}_{4} \mathrm{P}$

$N$-(1,11-Dioxa[11]paracyclophan-13-ylmethyl)- $N$-isopropyldibenzo[d,f][1,3,2]dioxaphosphepin-6-amine 
Madhurima Hazra, Kyalo Stephen Kanyiva, and Takanori Shibata

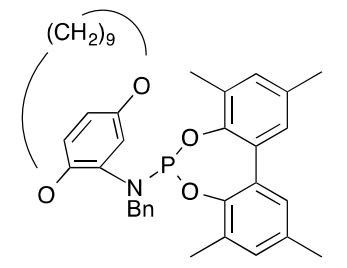

$[\alpha]_{\mathrm{D}}^{27}=-123.3^{\circ}\left(\right.$ c $\left.0.56, \mathrm{CHCl}_{3}\right)$

Source of planar chirality: $(+)$-sparteine

$\mathrm{C}_{38} \mathrm{H}_{44} \mathrm{NO}_{4} \mathrm{P}$

$N$-Benzyl- $N$-(1,11-dioxa[11]paracyclophan-13-yl)-2,4,8,10-tetramethyldibenzo[d,f][1,3,2]dioxaphosphepin-6-amine

Madhurima Hazra, Kyalo Stephen Kanyiva, and Takanori

Shibata

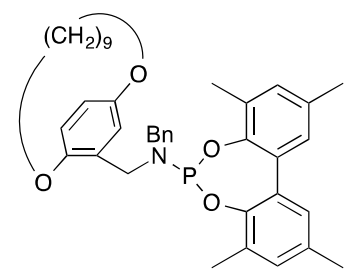

$[\alpha]_{\mathrm{D}}^{25}=+24.1^{\circ}\left(c 1.27, \mathrm{CHCl}_{3}\right)$

Source of planar chirality: $(+)$-sparteine

$\mathrm{C}_{39} \mathrm{H}_{46} \mathrm{NO}_{4} \mathrm{P}$

$N$-Benzyl- $N$-(1,11-dioxa[11]paracyclophan-13-ylmethyl)-2,4,8,10-tetramethyldibenzo[d,f][1,3,2]dioxaphosphepin-6-amine

Madhurima Hazra, Kyalo Stephen Kanyiva, and Takanori

Shibata
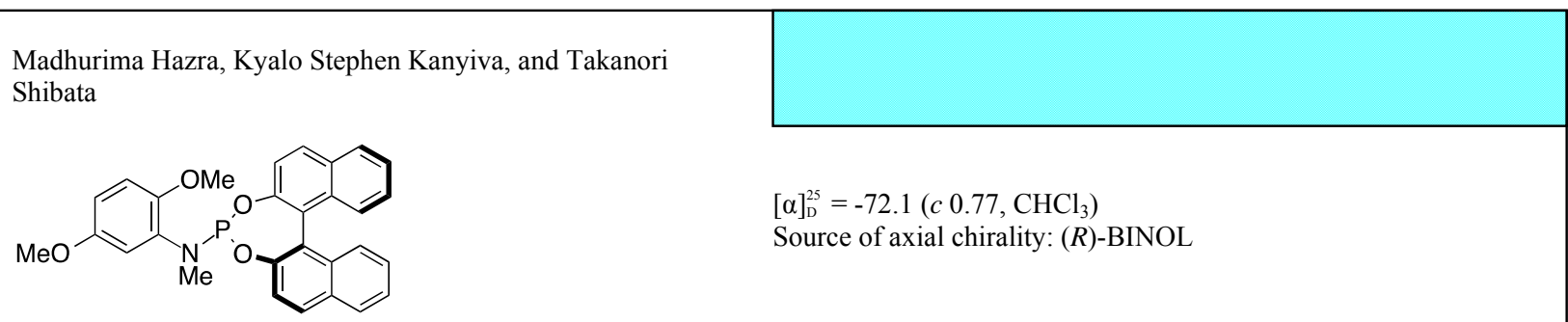

$[\alpha]_{\mathrm{D}}^{25}=-72.1\left(\right.$ c $\left.0.77, \mathrm{CHCl}_{3}\right)$

Source of axial chirality: $(R)-\mathrm{BINOL}$

$\mathrm{C}_{29} \mathrm{H}_{24} \mathrm{NO}_{4} \mathrm{P}$

$N$-(2,5-dimethoxyphenyl)- $N$-methyldinaphtho[2,1-d:1',2'-f][1,3,2]dioxaphosphepin-4-amine 


\section{Graphical Abstract}

To create your abstract, type over the instructions in the template box below.

Fonts or abstract dimensions should not be changed or altered.

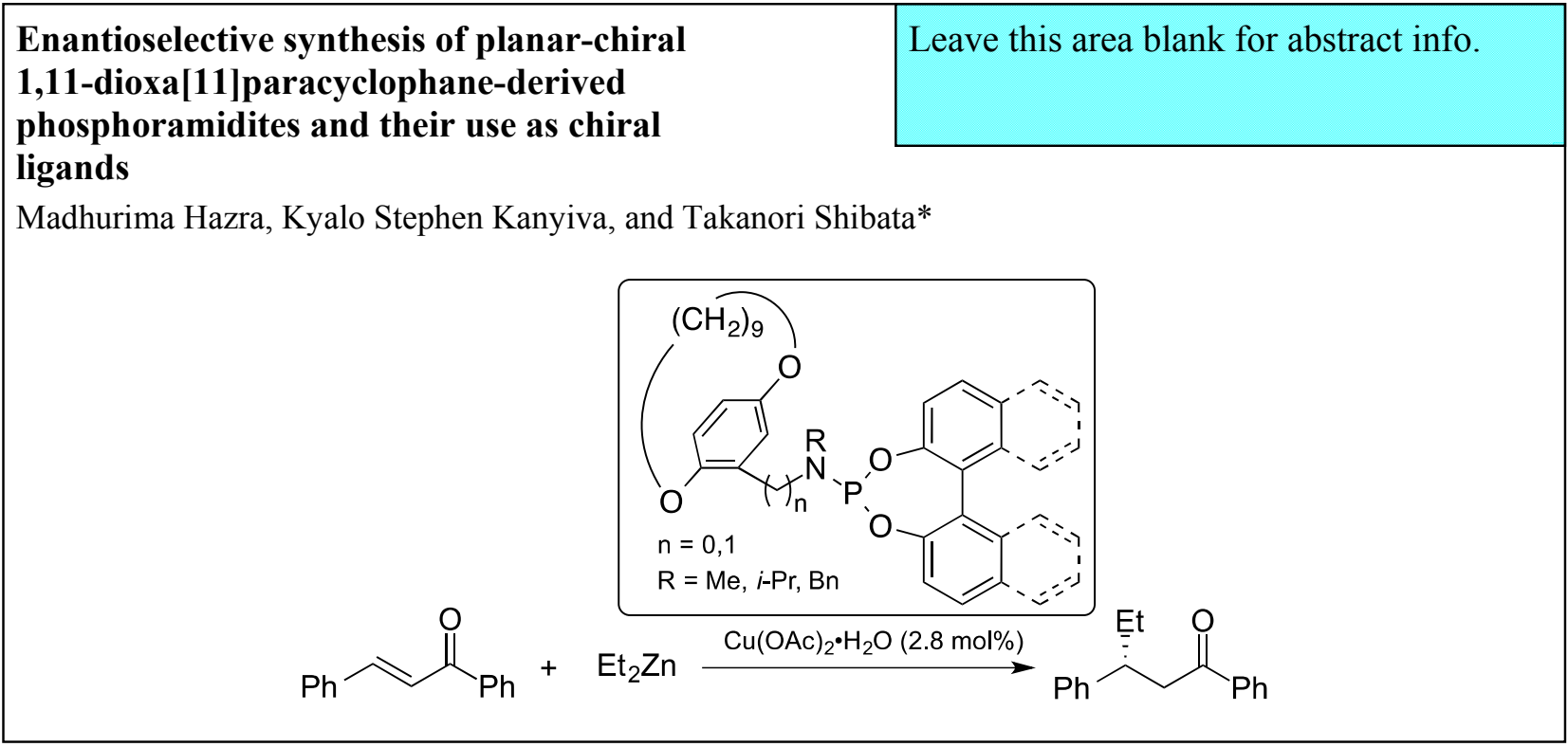

